\title{
Identification of DNA Methylation and Transcriptomic Profiles Associated With Fruit Mealiness in Prunus persica (L.) Batsch
}

OPEN ACCESS

Edited by:

Claudia Anabel Bustamante, Centro de Estudios Fotosintéticos y Bioquímicos (CEFOBI), Argentina

Reviewed by:

Pedro Martinez-Gomez, Center for Edaphology and Applied Biology of Segura, Spanish National

Research Council, Spain

George Manganaris,

Cyprus University of Technology,

Cyprus

Diep R. Ganguly,

Australian National University,

Australia

*Correspondence:

Claudio Meneses

claudio.meneses@unab.cl

Specialty section:

This article was submitted to

Plant Abiotic Stress,

a section of the journal

Frontiers in Plant Science

Received: 22 March 2021

Accepted: 17 May 2021

Published: 10 June 2021

Citation:

Rothkegel K, Espinoza A Sanhueza D, Lillo-Carmona V,

Riveros A, Campos-Vargas $R$ and Meneses C (2021) Identification of DNA Methylation and Transcriptomic Profiles Associated With Fruit Mealiness in Prunus persica (L.) Batsch.

Front. Plant Sci. 12:684130. doi: 10.3389/fpls.2021.684130

\section{Karin Rothkegel', Alonso Espinoza ${ }^{1}$, Dayan Sanhueza ${ }^{1}$, Victoria Lillo-Carmona', Anibal Riveros' ${ }^{1}$, Reinaldo Campos-Vargas ${ }^{2}$ and Claudio Meneses ${ }^{1,3 *}$}

${ }^{1}$ Facultad Ciencias de la Vida, Centro de Biotecnología Vegetal, Universidad Andrés Bello, Santiago, Chile, ${ }^{2}$ Departamento de Producción Agrícola, Facultad de Ciencias Agronómicas, Centro de Estudios Postcosecha, Universidad de Chile, Santiago, Chile, ${ }^{3}$ FONDAP Center for Genome Regulation, Santiago, Chile

Peach (Prunus persica) fruits have a fast ripening process and a shelf-life of days, presenting a challenge for long-distance consuming markets. To prolong shelf-life, peach fruits are stored at low temperatures $\left(0\right.$ to $\left.7^{\circ} \mathrm{C}\right)$ for at least two weeks, which can lead to the development of mealiness, a physiological disorder that reduces fruit quality and decreases consumer acceptance. Several studies have been made to understand this disorder, however, the molecular mechanisms underlying mealiness are not fully understood. Epigenetic factors, such as DNA methylation, modulate gene expression according to the genetic background and environmental conditions. In this sense, the aim of this work was to identify differentially methylated regions (DMRs) that could affect gene expression in contrasting individuals for mealiness. Peach flesh was studied at harvest time (E1 stage) and after cold storage (E3 stage) for 30 days. The distribution of DNA methylations within the eight chromosomes of $P$. persica showed higher methylation levels in pericentromeric regions and most differences between mealy and normal fruits were at Chr1, Chr4, and Chr8. Notably, differences in Chr4 co-localized with previous QTLs associated with mealiness. Additionally, the number of DMRs was higher in $\mathrm{CHH}$ cytosines of normal and mealy fruits at E3; however, most DMRs were attributed to mealy fruits from E1, increasing at E3. From RNA-Seq data, we observed that differentially expressed genes (DEGs) between normal and mealy fruits were associated with ethylene signaling, cell wall modification, lipid metabolism, oxidative stress and iron homeostasis. When integrating the annotation of DMRs and DEGs, we identified a CYP450 82A and an UDP-ARABINOSE 4 EPIMERASE 1 gene that were downregulated and hypermethylated in mealy fruits, coinciding with the colocalization of a transposable element (TE). Altogether, this study indicates that genetic differences between tolerant and susceptible individuals is predominantly affecting epigenetic regulation over gene expression, which could contribute to a metabolic alteration from earlier stages of development, resulting in mealiness at later stages. Finally, this epigenetic mark should be further studied for the development of new molecular tools in support of breeding programs.

Keywords: peach, chilling injury, Rosaceae, epigenetics, cytosine methylation 


\section{INTRODUCTION}

Peaches [Prunus persica (L.) Batsch] and nectarines [Prunus persica (L.) Batsch var. nectarina] are climacteric stone fruits that belong to the Rosaceae family. Once harvested, $P$. persica fruits ripen fast within days at room temperature in spring and summer. To slow down the ripening process and prolong postharvest life, a cold storage is usually used at $0{ }^{\circ} \mathrm{C}$ for at least two weeks. However, this method can trigger a physiological disorder known as chilling injury (CI), which is manifested during ripening (Lurie and Crisosto, 2005). The symptoms of CI include mealy texture, flesh browning and/or bleeding, and leatheriness. The main cause of mealiness has been attributed to the metabolism of cell wall pectins, where mealy fruits are associated with an altered pectin methylesterase (PME) and endo-polygalacturonase (PG) activity (Brummell et al., 2004). Concomitantly, it has been suggested that mealiness is caused by the accumulation of insoluble calcium-pectate gel complexes in the middle lamella, trapping free water (Ben-Arie and Lavee, 1971). Another reported effect of CI on P. Persica is a reduced cell-to-cell adhesion, resulting in cell separation instead of cell rupture when chewing (King et al., 1989). More recently, studies have also suggested an alteration in reactive oxygen species (ROS) metabolism, mitochondrial respiration, sugar catabolism, amino acid usage, proteomic reprogramming and the importance of jasmonic acid signals in reducing CI via ethylene and sugars (Kan et al., 2011; Monti et al., 2019; Zhao et al., 2021).

Mealiness is genetically influenced and is also related with maturity date, since early cultivars have a lower susceptibility than later cultivars (Crisosto et al., 1999). In 2015, Nuñez-Lillo et al. (2015) studied an F2 Venus $\times$ Venus population, detecting one QTLs (Quantitative Trait Loci) for mealiness on linkage group 4 (LG4), explaining $34 \%$ of phenotypic variation. Within those QTLs, nine candidate genes related to cell wall synthesis, ethylene signaling and cold stress were identified. Later in 2019, Nuñez-Lillo et al. (2019) studied an F1 O' Henry x NR-053 population, identifying an additional QTL in LG5, and an endo-1,3-beta-glucosidase as one of the candidate genes associated with mealiness.

At the transcriptomic level, Pons et al. (2014) studied a population (Pop-DG) segregating for $\mathrm{CI}$, indicating that the transcriptome of peach fruits was changing during cold storage and was concomitant with the development of CI, highlighting that genes associated with cold response were differentially regulated between sensitive and resistant individuals. Afterward, Pons et al. (2016), analyzed the transcript profiles of the same population, but with different cold storage treatments and subsequent ripening. The results showed that during cold storage, the ripening program involving ethylene, auxin, and cell wall metabolism became desynchronized in susceptible fruits. Another study using a tolerant "Oded" and a relatively tolerant "Hermoza" peach cultivars identified that genes related to oxidative stress and biosynthesis of metabolites with antioxidant activity were positively correlated with CI tolerance (Puig et al., 2015). Moreover, the results suggested that ethylene signaling is related to CI tolerance, but also that auxins may play a role.

To better understand the molecular mechanisms modulating gene expression during plant development and adaptation, epigenomic approaches are an important layer of information. Epigenetic modifications involve histone changes, non-coding RNAs and DNA methylation, which influence chromatin structure and accessibility to genetic information (Zhang et al., 2018). In particular, cytosine DNA methylation has a key role in development, imprinting, silencing of transposable elements, abiotic and biotic responses (Law and Jacobsen, 2010). DNA methylation can be present under three different cytosine contexts: $\mathrm{CpG}, \mathrm{CHG}$, and $\mathrm{CHH}$, where the $\mathrm{H}$ can be $\mathrm{C}, \mathrm{T}$, or A. An example of changes in DNA methylation during fruit ripening is the Colorless non-ripening (Cnr) mutant in tomato (Solanum lycopersicum), where colorless fruits loss cell-to-cell adhesion and have a mealy pericarp (Manning et al., 2006). This natural mutation involves high levels of cytosine methylation in a 286-bp region upstream of an SBPbox transcription factor, inhibiting fruit ripening and gene expression. A more recent study in tomato also showed that cytosine demethylation by a DNA glycosylase SIDML2/ROS1 is necessary for the activation of ripening-induced genes and inhibition of ripening-repressed genes, revealing a critical role for DNA methylation during fruit ripening (Lang et al., 2017). In addition, changes in DNA methylation can be further used for the identification of novel markers, which will consider the genetic background, together with changing environmental conditions, as recently studied in Prunus (Prudencio et al., 2018; Rothkegel et al., 2020). In this sense, molecular approaches associated with agronomical traits should be implemented together with classical genetics in order to support breeding programs (García-Gómez et al., 2021).

Mealiness is a well-studied disorder, however, the molecular mechanisms leading to this phenotype are not entirely known. In order to elucidate if changes in DNA methylation levels are involved with susceptibility to cold storage, the aim of this work was to identify differentially methylated regions (DMRs) that could affect gene expression in contrasting individuals for mealiness at harvest time and after cold storage. This work will contribute with the first epigenomic study on mealiness, novel DMRs and additional differentially expressed genes (DEGs) that could be further studied as potential markers to differentiate susceptible peach fruits from tolerant fruits at harvest time.

\section{MATERIALS AND METHODS}

\section{Plant Material and Phenotyping}

A previously characterized $\mathrm{F} 2 \mathrm{~V} \times \mathrm{V}$ population of 151 individuals [Prunus persica (L.) Batsch] was used for fruit quality characters obtained from the self-pollination variety "Venus" (Nuñez-Lillo et al., 2015; Lillo-Carmona et al., 2020). This population located at Institute of Agricultural Research (INIA), Rayentué, Región de O’Higgins, Chile $\left(34^{\circ} 32^{\prime} 14^{\prime \prime} \mathrm{S}\right.$, $\left.70^{\circ} 83^{\prime} 44^{\prime \prime} \mathrm{W}\right)$, corresponds to six-year-old trees grown on 
$\mathrm{G} \times \mathrm{N}$ rootstock that segregates for mealiness, soluble solids content and maturity date. These fruits are freestone, with yellow and melting flesh. For season 2016-2017, peach fruits were harvested and at least five fruits were evaluated considering one individual with tolerance to cold and another individual with susceptibility to develop mealiness as determined in previous seasons (2012, 2013, and 2014) by our laboratory (NuñezLillo et al., 2015; Lillo-Carmona et al., 2020). Fruits were harvested (E1 stage) and stored at $-80{ }^{\circ} \mathrm{C}$ considering flesh firmness of $53 \mathrm{~N}$ and $0.8-1.2$ values (average of each peach cheek measurement) of chlorophyll absorbance index $\left(I_{A D}\right)$ with a portable Vis/NIR DA-Meter. Flesh firmness was determined using a Fruit Pressure Tester (Effigi, Alfonsine, Italy) on both peach cheeks.

A second pool of harvested fruits was stored at $0{ }^{\circ} \mathrm{C}$ for 30 days, corresponding to E3 stage (Figure 1A). After cold storage, fruits were stored at $-80{ }^{\circ} \mathrm{C}$ until further use, while another group of fruits was stored at $20{ }^{\circ} \mathrm{C}$ for 7 days until flesh firmness was approximately $7 \mathrm{~N}$, corresponding to E4 stage (ready to eat). Fruits from each pool and condition were evaluated for juiciness considering at least five biological replicates (Figure 1A). For E4 stage, firmness and juiciness were evaluated, while for E3 stage only firmness was measured. Juiciness was determined through a protocol described by Infante et al. (2009), where fruits with a value lower than $30 \%$ of juice were referred as mealy fruits, whereas fruits with higher values were considered as normal fruits. These juiciness values were compared using a mean separation test (ANOVA) and post-hoc Tukey-HSD test $(P<0.05)$. Finally, three fruits per individual (normal and mealy) were used at E1 and E3 stages for MethylC-Seq and RNA-Seq.

\section{MethylC-Seq}

Bisulfite treatment was carried out from genomic DNA obtained from frozen peach flesh at harvest time (E1) and after cold storage (E3). Three fruits from a tolerant individual and additional three fruits from a sensitive individual were used as biological replicates. Genomic DNA was extracted using a DNeasy Plant mini kit (QIAGEN, Germantown, MD, United States) according to manufacturer's instructions. The integrity of DNA was assessed in a $1.5 \%(\mathrm{p} / \mathrm{v})$ agarose gel and concentration was determined with Qubit Fluorometer (Thermo Fisher Scientific, Waltham, MA, United States). Afterward, $100 \mathrm{ng}$ of DNA was used for bisulfite treatment with the EZ DNA methylation gold kit (Zymo, Irvine, CA, United States) as previously described (Rothkegel et al., 2017). Twelve bisulfite treated libraries were generated considering two individuals (normal and mealy), two sampling points (E1 and E3) and three biological replicates. Libraries were generated with the TruSeq DNA Methylation kit (Illumina, San Diego, CA, United States) according to manufacturer's instructions. All libraries were validated with Qubit Fluorometer and Fragment Analyzer (Advanced Analytical Technologies, Ankeny, IA, United States) and later sequenced in HiSeq 4000, $2 \times 100$ bp Paired-end mode (Illumina, San Diego, CA,
United States). Raw data is available in NCBI sequence read archives (PRJNA705001).

\section{Processing of MethylC-Seq Reads and Identification of Differentially Methylated Regions}

Initially, raw reads were filtered and adapters removed using Trim Galore ${ }^{1}$. Filtered reads were mapped against the reference genome of $P$. persica v.2.0.al using Bismark with no mismatches to obtain the methylation state of $\mathrm{CpG}, \mathrm{CHG}$, and $\mathrm{CHH}$ cytosine contexts (Krueger and Andrews, 2011; Verde et al., 2017). At the same time, reads were aligned to the plastid genome of $P$. persica, which is unmethylated, in order to obtain the bisulfite conversion rate. The bisulfite conversion rate was obtained by dividing the number of methylated cytosines and total cytosines of the chloroplast genome. For the identification of DMRs we used methylPipe considering cytosines covered with at least five reads and a $P$-value of 0.05 or less, a binsize of 10 and at least ten consecutives methylated cytosines using the find DMR function (Kishore et al., 2015). Comparisons were made for: (1) E3 vs. E1 in normal and mealy fruits; and (2) mealy vs. normal fruits at E1 and E3. A Kruskal-Wallis test was performed and regions with changes in methylation of at least two-fold were considered as DMR. Genes that overlapped with a DMR, including 2,000 bp upstream and downstream, were annotated as differentially methylated and used to perform a Gene Ontology (GO) analysis (FDR < 0.01) using AgriGO and REVIGO to decrease redundancy ${ }^{2}$.

\section{RNA-Seq}

Total RNA from 4 gr of frozen peach mesocarp was isolated using the sodium borate decahydrate method described by Meisel et al. (2005). RNA integrity was assessed using Fragment Analyzer electrophoresis and Qubit Fluorometer. Twelve strand-specific libraries (2 individuals $\times 2$ conditions $\times 3$ biological replicates), were generated from $1 \mu \mathrm{g}$ of total RNA using TruSeq Stranded mRNA kit (Illumina, San Diego, CA, United States). Validated libraries were sequenced in HiSeq 4000, $2 \times 100$ bp Paired-end mode and raw data is available in NCBI sequence read archive (PRJNA705416).

\section{Processing of RNA-Seq Reads and Identification of Differentially Expressed Genes}

Sequenced reads were trimmed with Trim Galore and mapped to the reference genome of $P$. persica v.2.0.al using Spliced Transcripts Alignment to a Reference (STAR; Dobin et al., 2013). Uniquely mapped reads were normalized as trimmed mean of M-values (TMM) and used for DEG analysis with EdgeR considering a False Discovery Rate $($ FDR $)<0.01$ and a foldchange of 1 or more (Robinson et al., 2010). Differentially

\footnotetext{
${ }^{1}$ https://www.bioinformatics.babraham.ac.uk/projects/trim_galore/

${ }^{2}$ http://bioinfo.cau.edu.cn/agriGO/analysis.php
} 


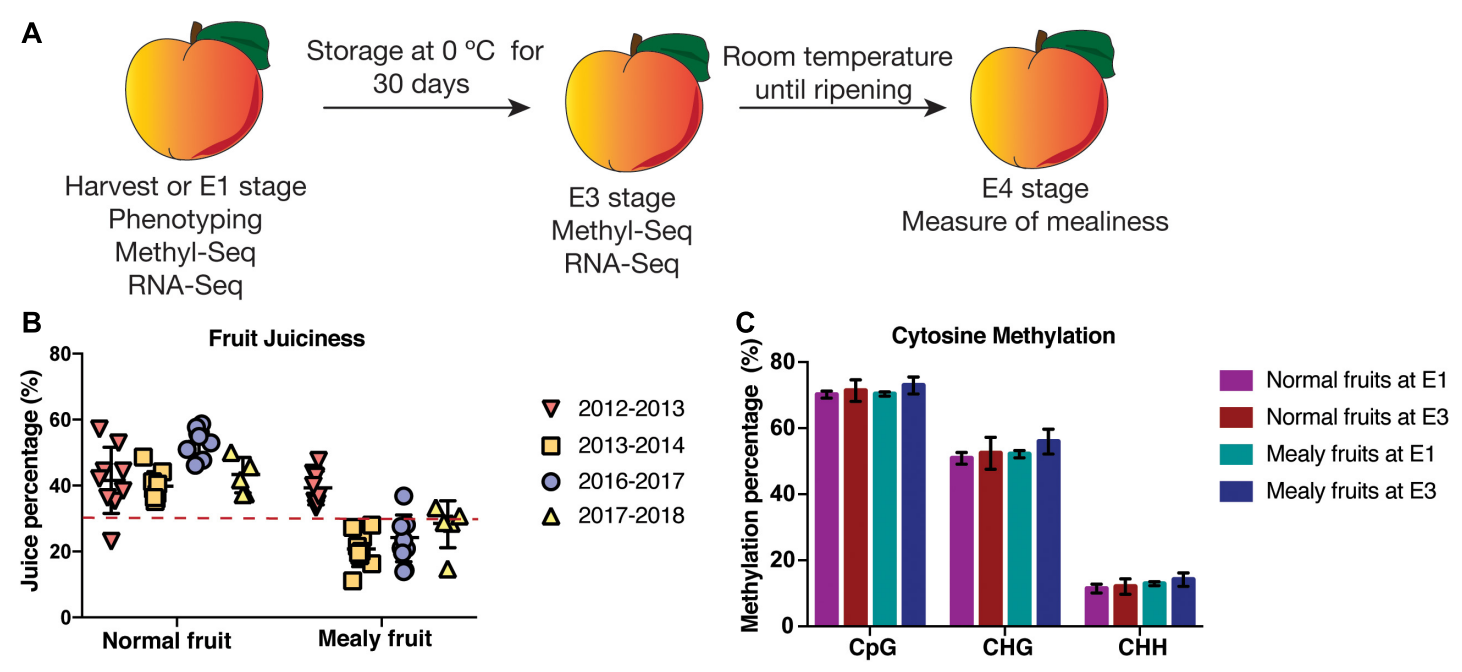

FIGURE 1 | Phenotyping and global levels of DNA methylation in P. persica fruits susceptible and resistant to mealiness. (A) Experimental design for one resistant and one susceptible individual for mealiness. At harvest (E1), three fruits per tree were used for MethylC-Seq and RNA-Seq analysis, while another pool of fruits was stored in a cold chamber at $0^{\circ} \mathrm{C}$ to promote mealiness. After 30 days of cold treatment (E3 stage), three fruits were used for MethylC-Seq and RNA-Seq analysis. At E4 stage, at least five fruits were ripened at room temperature and used for the measure of juice content. (B) Juice content (\%) during four seasons in at least five fruits of an individual resistant and susceptible to mealiness, respectively. The dotted line represents a $30 \%$ threshold to consider fruits as mealy or normal. The same trees were evaluated in all seasons. (C) Absolute methylation levels (\%) for cytosine contexts $\mathrm{CpG}, \mathrm{CHG}$ and $\mathrm{CH} H(\mathrm{H}=\mathrm{C}, \mathrm{T}$ or $\mathrm{A})$ in normal and mealy fruits at $\mathrm{E} 1$ and E3.

expressed genes were obtained by comparing (1) E3 vs. E1 in normal and mealy fruits; and (2) mealy vs. normal fruits at E1 and E3. Variability in gene expression between mealy and normal fruits at E1 and E3 was considered to rank the top50 genes whose expression level was affected the most, represented as a heatmap. Additionally, DEGs that overlapped with DMRs, including 2,000 bp upstream and downstream of gene annotation, were considered as differentially methylated. Hypermethylated regions associated with downregulated genes and vice-versa were represented as heatmaps considering expression levels.

\section{Real Time qPCR Analysis}

From total RNA, one microgram was treated with DNase I (Thermo Fisher Scientific), followed by the synthesis of cDNA using the SuperScript ${ }^{\mathrm{TM}}$ first-strand synthesis system and oligo dT primers (Thermo Fisher Scientific), according to the standard protocol. Before use, each cDNA sample was diluted 1:10 with nuclease free water. The RT-qPCR assay was performed in an AriaMx real-time PCR system (Agilent Technologies, Santa Clara, CA, United States) with a master mix of KAPA SYBR ${ }^{\circledR}$ FAST qPCR master mix (Kapa Biosystems, Wilmington, MA, United States), $10 \mu \mathrm{M}$ of forward and reverse primers (Supplementary Table 1), ROX dye, template cDNA and PCR-grade water for a final volume of $10 \mu \mathrm{L}$. All RT-qPCR assays were performed using three biological and three technical replicates. The expression profiles of PpeSAUR50 (Prupe.3G035000), PpeERF4 (Prupe.4G176200), PpeERF5 (Prupe.5G062000), and PpeXET (Prupe.3G172000) were normalized to PpeTEF2 (EST TC3544) (Tong et al., 2009).

\section{RESULTS}

\section{Methylome Sequencing of Nectarine Fruits Contrasting for Mealiness}

In order to determine the phenotypic parameters of mealiness, initially we used a $\mathrm{V} \times \mathrm{V}$ population of 151 siblings that was previously phenotyped during 2012, 2013, and 2014 for firmness, soluble solids contents, titratable acidity, weight and $I_{A D}$ at E1 stage (Nuñez-Lillo et al., 2015). During these seasons, the average juiciness was $32.6 \%$, ranging between 15 and $51 \%$ and obtaining a unimodal distribution. Afterward, during season 2016-2017, the same phenotypic parameters were measured, showing no significant differences for firmness, $I_{A D}$ and soluble solids content (Lillo-Carmona et al., 2020). Moreover, juiciness ranged from $21,56 \%$ in mealy fruits, to $52.68 \%$ in normal fruits.

For the present study, we used the same two contrasting siblings from season 2016 to 2017 phenotyped by Lillo-Carmona et al. (2020) since they showed a consistent phenotype for mealiness during at least two seasons. For flesh juiciness, fruits showing a $30 \%$ or less of juice content were considered as mealy, obtaining fruits with a normal juice content ( 44.5\%) during four seasons (2012-2013; 2013-2014; 20162017, and 2017-2018) and mealy fruits during three seasons (2013-2014; 2016-2017, and 2017-2018), showing a variable phenotype (Figure 1B).

Afterward, normal and mealy fruits were sequenced at E1 and E3 stages to analyze the methylation profiles that could be associated with the phenotype (Table 1). From total bisulfite reads, at least a $53.4 \%$ mapped uniquely to the Prunus persica v2.0 genome, whereas $17.2 \%$ reads showed no alignment. From uniquely mapped reads, approximately a $71.2 \%$ of cytosines were 
methylated in the CpG context, followed by a $52.8 \%$ in the $\mathrm{CHG}$ context and a $12.6 \%$ in the $\mathrm{CHH}$ context, with no significant differences between conditions (Figure 1C; Supplementary Table 2). At the same time, to analyze the bisulfite conversion rate, we mapped bisulfite reads to the chloroplast genome of peach, obtaining at least a $99.3 \%$ of conversion rate (Supplementary Table 3). To analyze the variability of global methylations, we conducted a PCA (Supplementary Figure 2). Despite PCA explains only a $25 \%$ of variability in DNA methylations, normal fruits at E1 and E3 are well separated from mealy fruits at the same conditions.

When studying the methylation level within the eight chromosomes of peach, we observed that highest levels of methylation are associated with the average location of centromeres. Differences between mealy and normal fruits were present in Chr1 with increased methylation in mealy fruits and Chr2 with increased methylation at 6,081,175 bp in normal fruits. Additionally, Chr4 showed higher methylation levels in mealy fruits, whereas Chr8 possess regions with higher methylation in mealy fruits and others with higher methylation in normal fruits (Figure 2A). Furthermore, methylation differences within Chr4 were mostly contributed to $\mathrm{CHG}$ and $\mathrm{CHH}$ cytosine contexts, which also co-localized with previously reported QTLs for mealiness (Figure 2B) (Cantín et al., 2010; Nuñez-Lillo et al., 2015, 2019).

The candidate genes contained within these QTLs located in Chr4 and Chr5 are shown in Table 2. From these genes, those that presented DMRs between mealy and normal fruits at E1 are associated with gibberellin biosynthesis, with an increase of $45.9 \%$ in the methylation level of mealy fruits, signal transduction with an increase of $9.9 \%$, protein transport with an increase of $4.9 \%$ and cell wall modification with a decrease of $-13.7 \%$. At E3, differences in methylation levels includes a gene related with carbohydrate transport, showing an increase of $46.6 \%$ in mealy fruits, genes related with protein phosphorylation $(-24.8 \%)$ and iron ion binding $(-10.8 \%)$ (Table 2).

\section{Annotation and Identification of Differentially Methylated Regions in Normal and Mealy Fruits at E1 and E3 Stages}

To evaluate the distribution of methylations in all the annotated genes of P. persica ( $n=26,873$ genes), we obtained the methylation pattern within coding regions, including 2-kb upstream and 2-kb downstream (Figures 3A,B). For the CG context, methylation levels are higher upstream and downstream the transcribed regions, but with lower levels within TSS (transcription start site) and TES (transcription end site). Normal fruits showed a higher level of methylation than mealy fruits, however, all samples decreased their methylation when exposed to cold (E3 stage). On the contrary, for transposable elements (TE), CG methylations are higher within the TE rather than upstream and/or downstream. In the CHG context, methylation level is higher in upstream and downstream regions, maintaining low levels within transcribed regions. In the same context, TEs showed high levels of methylation, with a similar profile than CG methylations. In the $\mathrm{CHH}$ context, low levels of methylation are observed within the transcribed regions of genes, while high levels are present upstream and downstream. Regarding TEs, high levels of $\mathrm{CHH}$ methylation are observed within $\mathrm{TE}$, but higher levels are present in mealy fruits at E1 and E3 stages when compared to normal fruits.

For the identification of DMRs, we considered the bisulfite conversion rate of each sample and a $p$-value $<0.05$ for binominal test. When studying normal fruits at E3 vs. E1, we observed a similar amount of hypermethylated and hypomethylated regions, with most DMRs occurring in $\mathrm{CHH}$ context (Figure 3C). However, in mealy fruits at E3 vs. E1, $\mathrm{CHH}$ methylation showed higher number of hypermethylations ( $n=218$ DMRs) when compared to hypomethylations ( $n=119$ DMRs). When comparing mealy fruits vs. normal fruits at E1, we observed that differences in methylation mainly occurred in the $\mathrm{CHH}$ context with 274 hypermethylations and 167

TABLE 1 | Parameters of Prunus persica whole genome bisulfite sequencing and mapping of each sample and condition.

\begin{tabular}{|c|c|c|c|c|c|}
\hline Sample & Sequence pairs analyzed & Uniquely mapped reads & Multiple mapped reads & Reads with no alignments & Genome coverage \\
\hline Normal fruit at E1 R1 & $29,593,511$ & $15,855,721$ & $8,087,885$ & $5,649,905$ & $7 X$ \\
\hline Normal fruit at E1 R2 & $27,418,488$ & $14,642,251$ & $8,066,107$ & $4,710,130$ & $7 X$ \\
\hline Normal fruit at E1 R3 & $32,698,148$ & $17,503,344$ & $9,179,958$ & $6,014,846$ & $8 X$ \\
\hline Normal fruit at E3 R1 & $29,572,246$ & $15,849,712$ & $8,165,217$ & $5,557,317$ & $7 X$ \\
\hline Normal fruit at E3 R2 & $37,201,194$ & $19,621,951$ & $11,453,156$ & $6,126,087$ & $9 \times$ \\
\hline Normal fruit at E3 R3 & $39,364,014$ & $20,005,394$ & $13,327,321$ & $6,031,299$ & $9 x$ \\
\hline Mealy fruit at E1 R1 & $30,242,762$ & $15,788,636$ & $8,575,159$ & $5,878,967$ & $7 X$ \\
\hline Mealy fruit at E1 R2 & $29,659,178$ & $15,381,741$ & $8,749,903$ & $5,527,534$ & $7 X$ \\
\hline Mealy fruit at E1 R3 & $32,770,016$ & $15,350,153$ & $11,278,650$ & $6,141,213$ & $7 X$ \\
\hline Mealy fruit at E3 R1 & $43,879,328$ & $24,399,996$ & $13,014,693$ & $6,464,639$ & $11 X$ \\
\hline Mealy fruit at E3 R2 & $36,227,923$ & $19,872,745$ & $10,317,302$ & $6,037,876$ & $9 x$ \\
\hline Mealy fruit at E3 R3 & $35,383,415$ & $18,463,308$ & $11,327,215$ & $5,592,892$ & $8 x$ \\
\hline
\end{tabular}

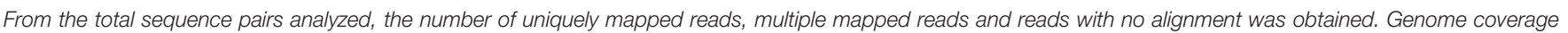

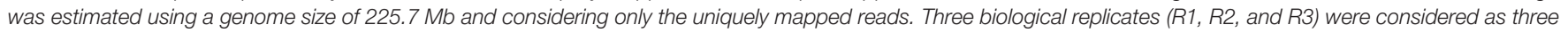
independent fruits per condition. 

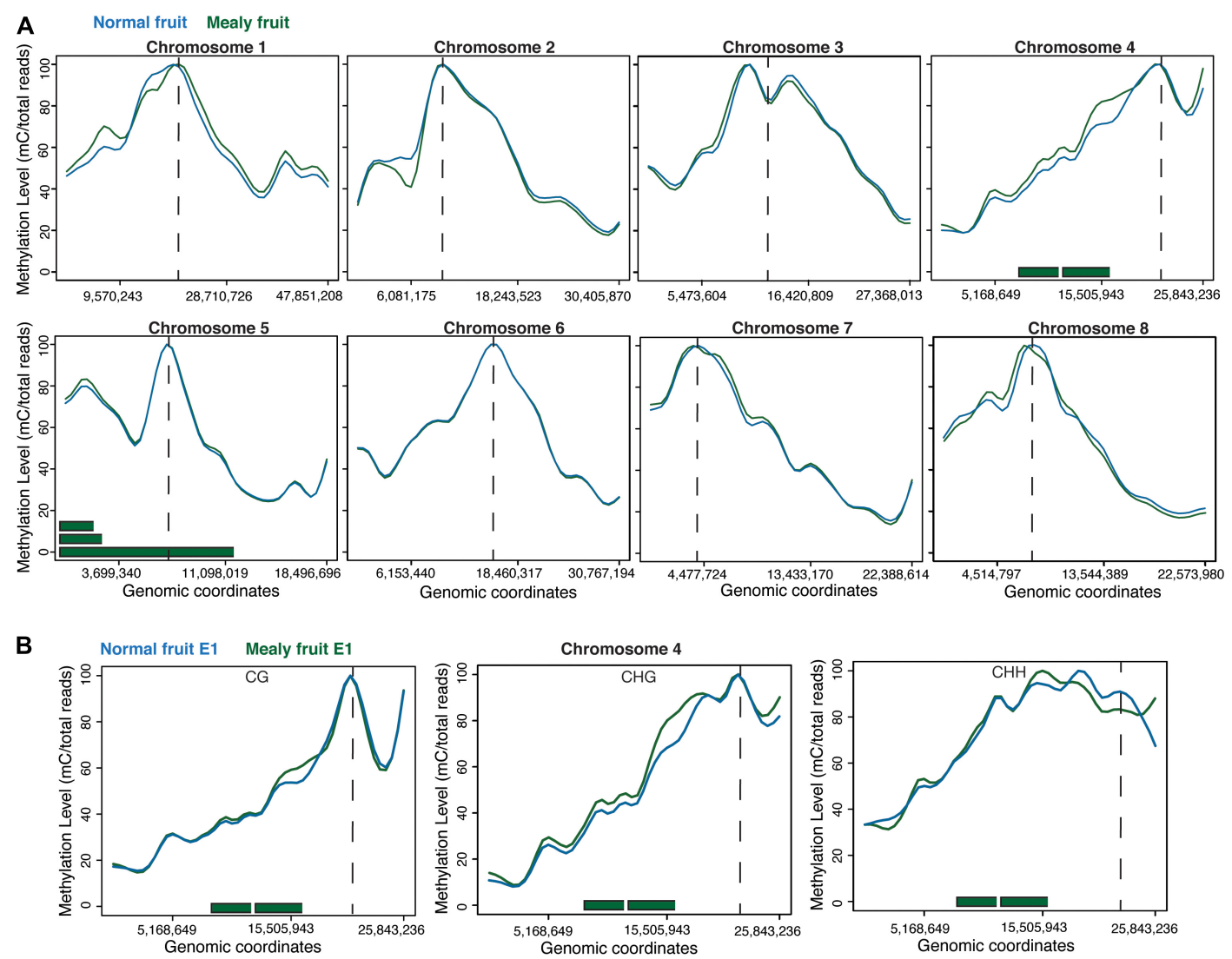

FIGURE 2 | DNA methylation levels across the eight pseudomolecules of $P$. Persica. (A) Methylation levels considering all cytosine contexts in normal fruits (blue line) and mealy fruits (green line). Horizontal green bars represent the location of previously identified QTLs for mealiness, while dashed vertical lines indicates the approximate location of centromeres. (B) Methylation levels in $\mathrm{CpG}, \mathrm{CHG}$, and $\mathrm{CHH}$ contexts within chromosome 4, the main candidate associated with mealiness phenotype based on QTL studies.

TABLE 2 | Genes that co-localized with QTLs for mealiness within chromosomes 4 and 5 and presented differentially methylated regions (DMRs) between mealy and normal fruits at $\mathrm{E} 1$ and $\mathrm{E} 3$ stages.

\begin{tabular}{lccccc}
\hline Stage & Gene ID & Description & Pathway & Methylation difference (\%) & Location \\
\hline E1 & Prupe.4G128700 & ENT-KAURENE SYNTHASE & Gibberellin biosynthesis & 45.9 & Intron \\
& Prupe.4G241500 & Mitochondrial import inner membrane translocase TIM50 & Protein transport & Downstream \\
& Prupe.5G074600 & Disease resistance protein-related & Signal transduction & Upstream \\
& Prupe.5G069600 & PECTINESTERASE 68-RELATED & Cell wall modification & -13.9 & Intron \\
E3 & Prupe.4G155700 & Bidirectional sugar transporter SWEET2 & Carbohydrate transport & 46.6 & Upstream \\
& Prupe.4G195300 & G-type lectin S-receptor-like serine & Protein phosphorylation & -24.8 & -10.8 \\
& Prupe.5G077800 & CYTOCHROME P450-LIKE PROTEIN RELATED & Iron ion binding & Intron \\
\hline
\end{tabular}

The methylation difference indicates an increase (positive) or decrease (negative) of methylation percentage in mealy fruits regarding to normal fruits.

hypomethylations in mealy fruits. Additionally, at E3, the number of hypermethylations in mealy fruits increased to 335 DMRs, while hypomethylations are maintained in 164 DMRs (Figure 3C). The annotation, methylation differences and description of each DMR in all cytosine contexts from normal fruits at E3 vs. E1, mealy fruits at E3 vs. E1, mealy fruits vs. normal fruits at E1 and E3 are detailed in Supplementary Tables 4-7, respectively.

From annotated DMRs, our interest was focused on which locations are more susceptible to change their methylation level. We observed that in normal fruits at E3, differences in methylation occurred upstream ( $n=11$ DMRs) of genes (Figure 4A), while in mealy fruits the number of DMRs is higher upstream ( $n=16$ DMRs) and downstream ( $n=22$ DMRs $)$ of genes (Figure 4B). On the other hand, in mealy fruits vs. normal fruits, the number of DMRs located upstream of genes increased from 37 DMRs at E1, to 46 DMRs at E3 (Figures 4C,D).

To better understand which pathways may be affected by these changes in DNA methylation, we performed a GO analysis of the annotated DMRs between mealy and normal fruits 


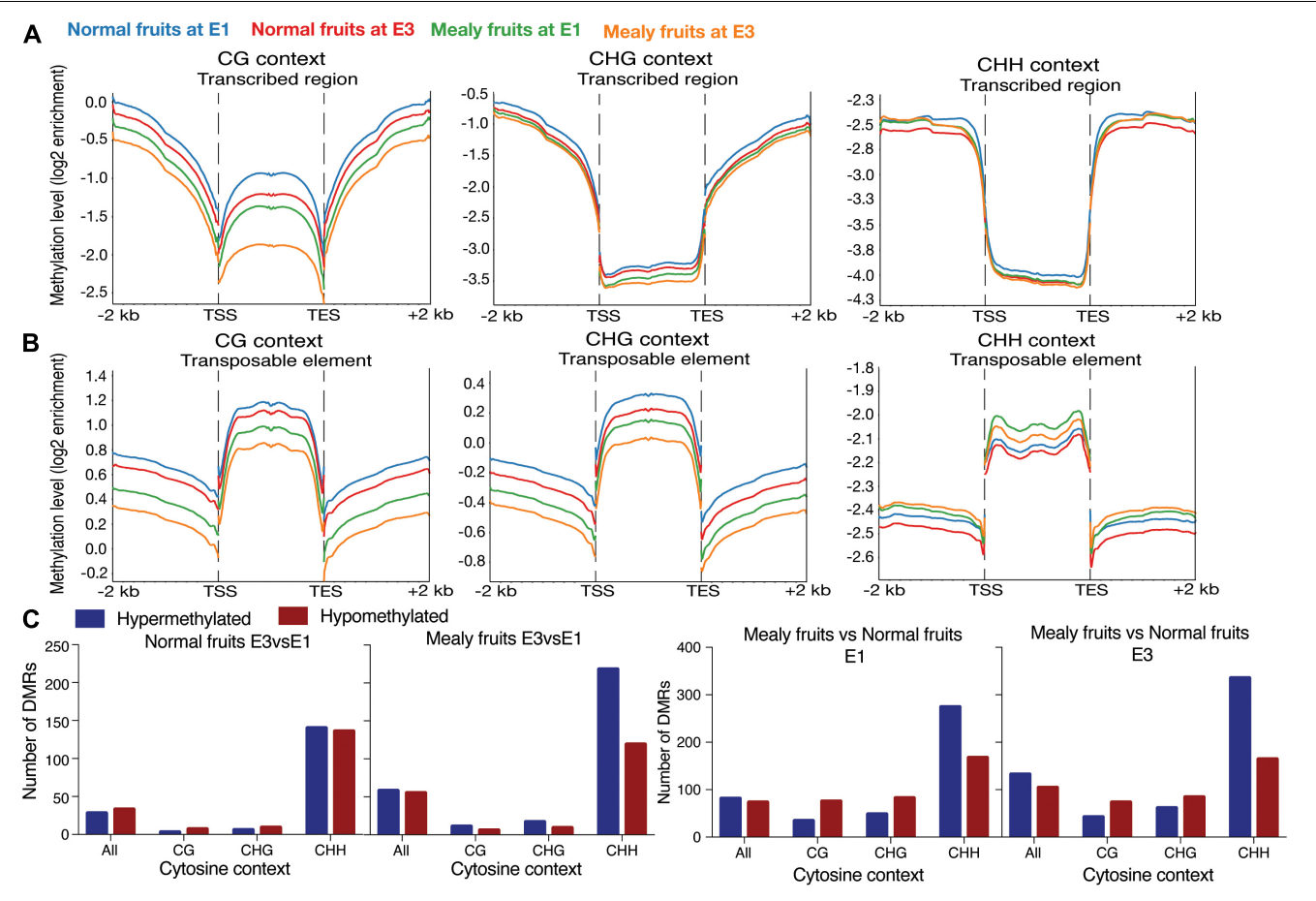

FIGURE 3 | Average distribution of global DNA methylation throughout coding regions and number of differentially methylated regions. (A) DNA methylation level in annotated genes, including $2 \mathrm{~kb}$ upstream and downstream of the transcription start site (TSS) and transcription end site (TES), respectively. Each color represents a different condition. (B) DNA methylation level in annotated transposable elements (TE), including $2 \mathrm{~kb}$ upstream and downstream of TSS and TES. (C) Number of Differentially Methylated Regions (DMRs) close to annotated genes (up to $2 \mathrm{~kb}$ upstream and downstream) in normal fruits at E3 vs. E1 stages, mealy fruits at E3 vs. E1, mealy fruits vs. normal fruits at E1 and mealy fruits vs. normal fruits at E3. Blue bars indicate hypermethylated regions and red bars hypomethylated regions. DMRs where considered in all cytosine contexts and individual contexts ( $\mathrm{CpG}, \mathrm{CHG}$, and $\mathrm{CHH}$ ). Three biological replicates, a log2 enrichment difference $>1$ and a $p$-value $<0.05$ were considered to identify a DMR.

(Table 3), where overrepresented molecular functions affected in mealy fruits corresponded to "ATP binding" and "purine nucleotide binding" at E1. At E3 stage, we observed an increase in overrepresented terms, where a higher frequency was obtained in "catalytic activity" with a $65.83 \%$, "ATP binding" with $14.13 \%$ and "purine nucleotide binding" with $16.11 \%$.

\section{Transcriptome Sequencing and Identification of Differentially Expressed Genes in Normal and Mealy Fruits}

In order to elucidate the transcriptional changes associated with the pattern of DNA methylations in response to chilling injury or mealiness, we analyzed RNA-Seq libraries from the same samples as MethylC-Seq (Table 4). Using samples from normal and mealy fruits at E1 and E3 stages, we obtained a 78-95\% of uniquely mapped reads, $1.5-5.5 \%$ of reads that aligned in multiple regions and $1.5-19.6 \%$ of reads that did not align to the reference of $P$. persica genome v2.0. Considering only the reads that aligned uniquely, we followed further analysis.

To visualize the distribution of global transcriptomic data, we used a PCA (Figure 5A). In this case, all the conditions (normal fruit at E1, normal fruit at E3, mealy fruit at E1, and mealy fruit at E3) are well separated, while biological replicates grouped together, explaining a $92 \%$ of variability. For the identification of DEGs, we first considered normal and mealy fruits at E3 regarding to $\mathrm{E} 1$, and additionally compared mealy fruits vs. normal fruits at E1 and E3 (Figure 5B). For the first comparison, we obtained a higher number of downregulated genes ( $n=3,436$ DEGs) in normal fruits and in mealy fruits ( $n=3,704$ DEGs) at E3. However, when comparing mealy fruits vs. normal fruits, we observed a decreased number of DEGs, where most of them are overexpressed in mealy fruits at E1 ( $n=966$ DEGs), increasing toward E3 ( $n=1,276$ DEGs). The annotation and transcript level of each DEG is detailed in Supplementary Tables 8-11.

Afterward, we ranked the top 50 DEGs with higher variance between normal and mealy fruits (Figure 5C). Genes that showed an increased expression in mealy fruits at E1 includes ETHYLENE-RESPONSIVE TRANSCRIPTION FACTORS (ERFs) 109 and 017, a cold acclimation gene $D R E B 1 D$, defense genes BON1 and WRKY70, and a cell wall gene GALACTURONOSYLTRANSFERASE-like 10. Another group of genes that showed an increased expression only in mealy fruits at E3 considers a lipid transport gene LTPG1, cell wall genes XYLOGLUCAN XTH8 and a probable POLYGALACTURONASE, whereas a ROS scavenging gene L-ASCORBATE PEROXIDASE APX2 was highly expressed both at E1 and E3 in mealy fruits. Genes that showed a decreased expression only in mealy fruits at E1 and E3 are ERF1B, a CELL WALL-ASSOCIATED RECEPTOR 


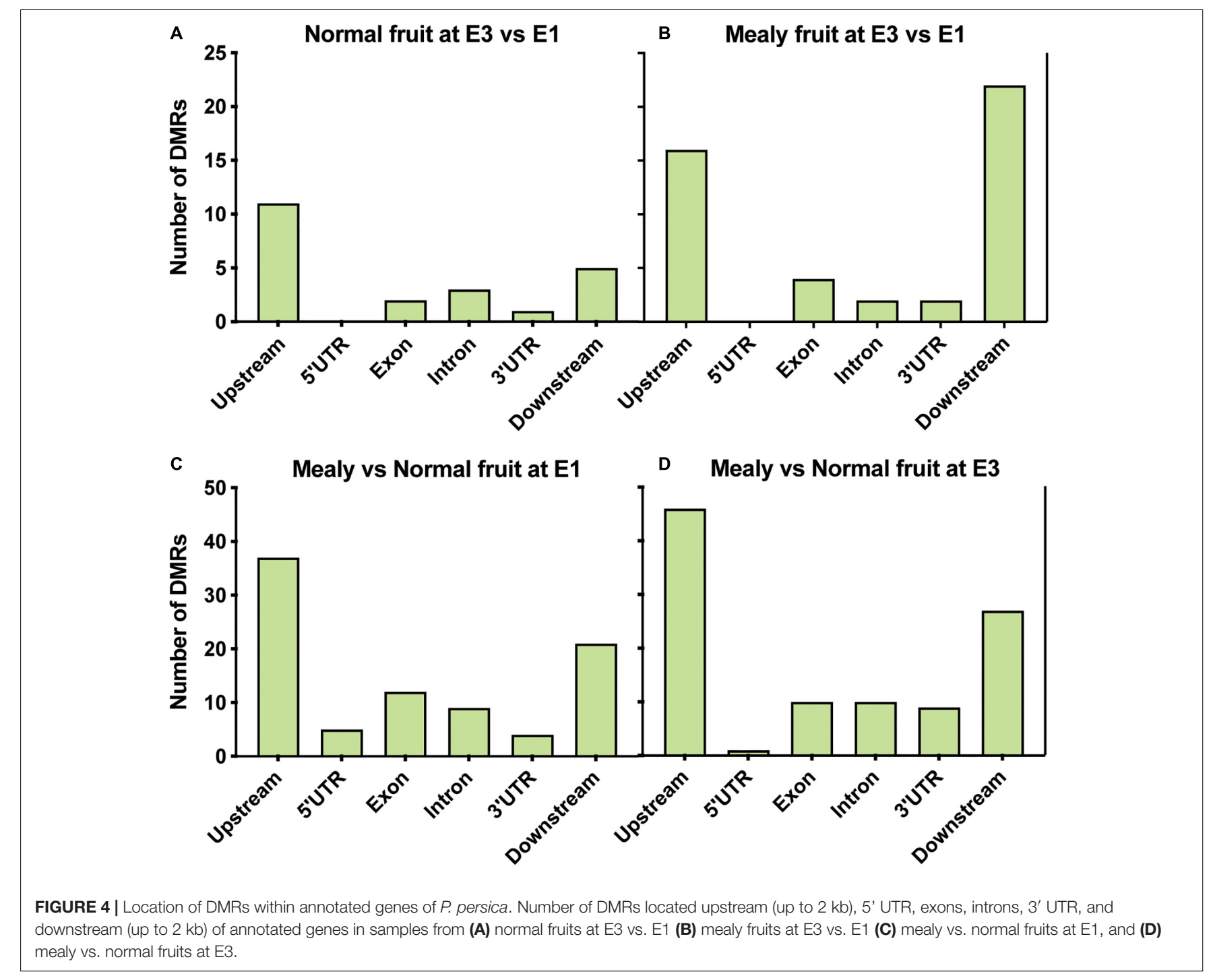

KINASE 2 WAK2, a UDP-GLYCOSYLTRANSFERASE, a GDSL esterase/lipase and a O-ACYLTRANSFERASE WSD1 gene from the lipid metabolism, an ARG7-like gene associated with auxin and cold response, and a CYTOCHROME P450 82A3 (CYP82A3) gene involved in sideretin biosynthesis in response to iron deficiency.

Additionally, in order to validate RNA-Seq data, we studied with real-time qPCR analysis the expression level of PpeSAUR50, PpeERF5, PpeERF4, and PpeXET, which were differentially expressed from RNA-Seq data between normal and mealy fruits (Supplementary Figure 2). The expression level of the four genes was similar between RNA-Seq and qPCR, showing differences between normal and mealy fruits.

\section{Integration of Methylomic and Transcriptomic Data}

For the integration of both methylation and transcriptomic data, we further studied only DMRs that co-localized with DEGs between normal and mealy fruits. At E1, hypermethylated genes that decreased their expression in mealy fruits correspond to a THREONINE-tRNA LIGASE and the recently mentioned CYP82A3 (Figure 6A). On the contrary, hypomethylated genes

TABLE 3 | Gene Ontology (GO) analysis of annotated genes associated with DMRs from mealy fruits vs. normal fruits at E1 and E3 stages.

\begin{tabular}{llcl}
\hline Stage & Molecular function & Frequency & Term ID \\
\hline E1 & ATP binding & $14.13 \%$ & GO:0005524 \\
& Purine nucleotide binding & $16.11 \%$ & GO:0017076 \\
E3 & Structural constituent of ribosome & $2.68 \%$ & GO:0003735 \\
& Catalytic activity & $65.83 \%$ & GO:0003824 \\
& Structural molecule activity & $3.27 \%$ & GO:0005198 \\
& ATP binding & $14.13 \%$ & GO:0005524 \\
& Ligase activity & $3.54 \%$ & GO:0016874 \\
& Purine nucleotide binding & $16.11 \%$ & GO:0017076
\end{tabular}

The columns show the overrepresented molecular functions, their frequency in each stage and the associated identity. 
that increased their expression in mealy fruits involves a CELLULOSE SYNTHASE-LIKE PROTEIN E6 and a probable GLUCOSE TRANSPORTER 3. Regarding to CYP82A3, the gene viewer of DNA methylations in mealy fruits at E1 and E3 revealed an hypermethylation across the entire gene located within the anti-sense strand. Furthermore, this hypermethylation is associated with the silencing of CYP82A3 and with the presence of a TE within the sense strand (Figure 6B). Additional hypermethylated genes at E3 that decreased their expression in mealy fruits includes an UDP-ARABINOSE 4EPIMERASE 1, FRIGIDA-like PROTEIN 3, a REGULATOR OF TELOMERE ELONGATION HELICASE 1, and a TMV RESISTANCE PROTEIN $N$ (Figure 6C). The methylation profile of UDP-ARABINOSE 4-EPIMERASE 1 also shows an hypermethylation upstream the gene, only in mealy fruits, which co-localized with a TE that may affect this pattern (Figure 6D).

Regarding to the higher methylation levels observed in mealy fruits, we further wanted to know if DNA methyltransferases and DNA demethylases changed their expression level in fruits susceptible to mealiness. For this, we searched for Arabidopsis thaliana orthologous genes within the P. Persica genome. Four putative DNA methyltransferases were identified in peach, one METHYLTRANSFERASE 1 (MET1), one CHROMOMETHYLTRANSFERASE 3 (CMT3) and two DOMAINS REARRANGED METHYLTRANSFERASE 2 (DRM2). Regarding to DNA demethylases, we identified one REPRESSOR OF SILENCING 1 (ROS1), one TRANSCRIPTIONAL ACTIVATOR DEMETER (DME) and one DEMETER-like 2 (DML2). The expression profile of each gene is shown in Figure 7. For the expression level of DNA methyltransferases, in general all of them decrease their expression after cold storage, however, only DRM2.2 shows significant differences between mealy and normal fruits, where mealy fruits presented a lower expression of DRM2.2 (Figure 7A). On the other hand, DNA demethylases ROS1 and DME showed a significant downregulation in mealy fruits at E3 (Figure 7B).

\section{DISCUSSION}

\section{The Methylome of $P$. persica Fruits Reveals Epigenomic Differences Between Normal and Mealy Fruits Associated With Genetic Variance}

Mealiness is a texture disorder characterized by the lack of juice from the flesh. In this study, we used samples from season 2016 to 2017, where no significant differences were obtained in firmness, $I_{A D}$ and soluble solids (Lillo-Carmona et al., 2020). However, for fruit juiciness mealy fruits presented a $21.56 \%$ of juice and normal fruits a $52.68 \%$, coincident with this textural disorder. Although mealiness is mostly influenced by the genetic background and maturation stage of the fruit, there is also an environmental factor that causes seasonal variability within the phenotype (Campos-Vargas et al., 2006), reason why a tree can produce mealy or normal fruits according to the season, as observed in Figure 1B. In fact, the contribution of the environmental effect over mealiness variance has been estimated between 36.5 and $57.5 \%$ in biparental populations of peach, coinciding with correlation values between seasons (Peace et al., 2005; Cantín et al., 2010; Nuñez-Lillo et al., 2015). Together with this, PCA results explained a $25 \%$ of variability in DNA methylations between normal and mealy fruits, indicating that most differences in global DNA methylation are due to genetic differences between siblings and to a lesser extend due to cold temperatures.

The methylome of nectarine fruits, obtained from two individuals with contrasting susceptibility to mealiness, was studied through MethylC-Seq before cold storage (E1 stage) and after 30 days at $0{ }^{\circ} \mathrm{C}$ (E3 stage). From methylome results, both in normal and mealy fruits, cytosine methylation was higher in the $\mathrm{CpG}$ context, followed by $\mathrm{CHG}$ and $\mathrm{CHH}$, coinciding with previous methylome studies of Arabidopsis thaliana, apple (Malus domestica borkh.), Populus euphratica and sweet cherry (Prunus avium L.), showing that different pathways regulate DNA methylation (Lister et al., 2008; Daccord et al., 2017; Su et al., 2018; Rothkegel et al., 2020).

TABLE 4 | Parameters of Prunus persica transcriptome sequencing (RNA-Seq) and mapping of each sample and condition.

\begin{tabular}{|c|c|c|c|c|c|}
\hline Sample & Processed reads & Uniquely mapped reads & Multiple mapped reads & Reads with no alignments & GC (\%) \\
\hline Normal fruit at E1 R1 & $26,432,188$ & $22,634,900$ & 703,271 & $3,094,017$ & 47 \\
\hline Normal fruit at E1 R2 & $36,943,878$ & $28,957,820$ & 726,711 & $7,259,347$ & 48 \\
\hline Normal fruit at E1 R3 & $32,478,417$ & $26,966,410$ & 635,225 & $4,876,782$ & 48 \\
\hline Normal fruit at E3 R1 & $26,658,213$ & $24,597,488$ & $1,145,517$ & 915,208 & 47 \\
\hline Normal fruit at E3 R2 & $20,609,623$ & $19,335,278$ & 840,540 & 433,805 & 46 \\
\hline Normal fruit at E3 R3 & $29,430,088$ & $26,858,876$ & $1,277,757$ & $1,293,455$ & 47 \\
\hline Mealy fruit at E1 R1 & $24,835,025$ & $20,663,761$ & 381,677 & $3,789,587$ & 48 \\
\hline Mealy fruit at E1 R2 & $30,053,367$ & $28,573,366$ & 572,053 & 907,948 & 47 \\
\hline Mealy fruit at E1 R3 & $29,446,889$ & $27,909,819$ & 526,394 & $1,010,676$ & 47 \\
\hline Mealy fruit at E3 R1 & $76,758,936$ & $70,198,217$ & $3,842,565$ & $2,718,154$ & 46 \\
\hline Mealy fruit at E3 R2 & $83,076,369$ & $77,232,835$ & $4,515,452$ & $1,328,082$ & 46 \\
\hline Mealy fruit at E3 R3 & $104,224,838$ & $92,005,533$ & $5,829,593$ & $6,389,712$ & 47 \\
\hline
\end{tabular}

Three biological replicates were considered as three independent fruits. 

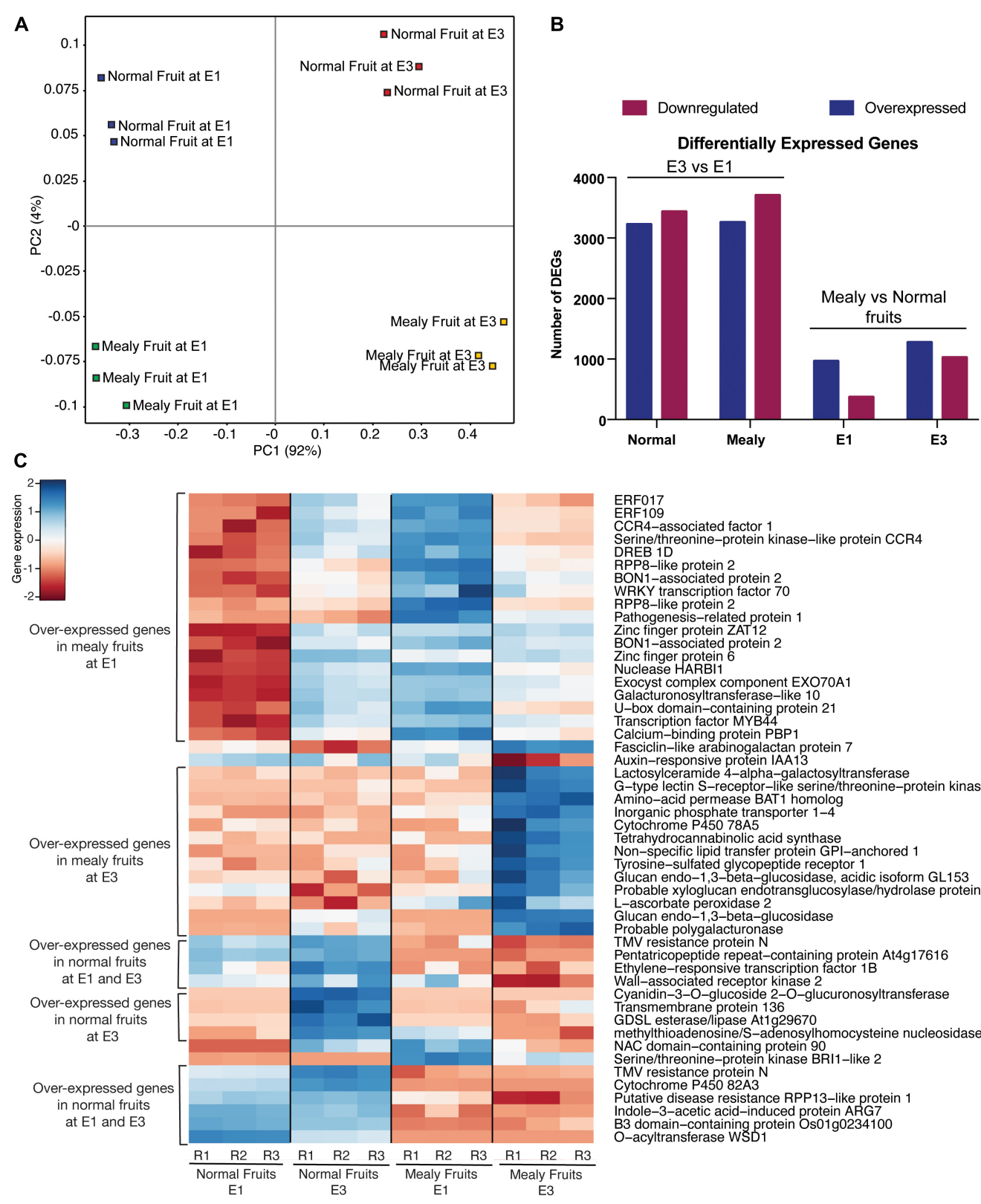

Differentially Expressed Genes

(1)

ERFF17

CCR 4-associated factor 1

Serine/threonine-protein kinase-like protein CCR4

RPP8-like protein 2

BON1-associated protein 2

WRKY transcription factor 70

RPP8-like protein 2

Pathogenesis-related protein
Zinc finger protein ZAT12

BON1-associated protein 2

Zinc finger protein 6

Exocyst complex component EXO70A1

Galacturonosyltransferase-like 10
U.box domain-containing protein 21

Transcription factor MYB 44
Calcium-binding protein PBP1

Calcium-binding protein PBP1
Fasciclin-like arabinogalactan protein 7

Auxin-responsive protein IAA13
Lactosylceramide 4-alpha-galactosyltransferas

G-type lectin S-receptor-like serine/threonine-protein kinase

Amino-acid permease BAT1 homolog
Inorganic phosphate transporter 1-4

Inorganic phosphate transporter 1-4

Cytochrome P450 78A5

Tetrahydrocannabinolic acid synthase
Non-specific lipid transfer protein GPI-anchored 1

Tyrosine-sulfated glycopeptide receptor 1

Glucan endo-1,3-beta-glucosidase, acidic isoform GL153

Probable xyloglucan endotransglucosylase/hydrolase protein 8

Gascorbate peroxidase 2

(a)

Trobable polygalacturonase
TMV resistance protein N

Pentatricopeptide repeat-containing protein At4g17616

Ethylene-responsive transcription factor $1 \mathrm{~B}$

Wall-associated receptor kinase

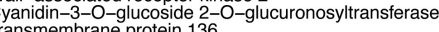

GDSL esterase/lipase At1929670

methylthioadenosine/S-adenosylhomocysteine nucleosidase 1

NAC domain-

Serine/threonine-protein kinase BRI1-like 2

TMV resistance protein $\mathrm{N}$
Cytochrome P $45082 \mathrm{A3}$

Putative disease resistance RPP13-like protein 1

Indole-3-acetic acid-induced protein ARG7

B3 domain-containing protein Os01g0234100

E1

FIGURE 5 | Transcriptomic profile of P.persica fruits susceptible and resistant to mealiness. (A) Principal Component Analysis (PCA) of global gene expression in normal (resistant) and susceptible fruits at E1 and E3. (B) Number of differentially expressed genes (DEGs) at E3 vs. E1, and in mealy vs. normal fruits. (C) Heatmap of the top 50 DEGs with higher variance in their transcript level between normal fruits and mealy fruits. Color key indicates the median log2 TMM-normalized values, where red to blue represents low to high levels of transcript, respectively.

At the chromosome level, regions with higher methylation indicate the approximate location of centromeres, which are mainly comprised by TEs and repetitive elements (Verde et al., 2013). Therefore, methylation in all cytosine context is higher in pericentromeric heterochromatin (Lister et al., 2008). Additionally, differences in methylation between mealy and normal fruits within Chr1, Chr2, Ch4, and Chr8 could be attributed to the genetic variance and polymorphisms between individuals, affecting the methylation pattern. For instance, higher methylation levels within Chr4 of mealy fruits are close to pericentromeric heterochromatin and also co-localized with previously identified QTLs for mealiness, confirming genetic variability (Nuñez-Lillo et al., 2015).

Candidate genes for mealiness comprised within QTLs (Nuñez-Lillo et al., 2015; Nuñez-Lillo et al., 2019), also presented DMRs between mealy and normal fruits. At E1 stage, an increase in methylation of mealy fruits was observed in ENT-KAURENE SYNTHASE, a gene involved in gibberellin biosynthesis, a plant growth phytohormone suggested to reduce mealiness in nectarine fruits when applied exogenously 

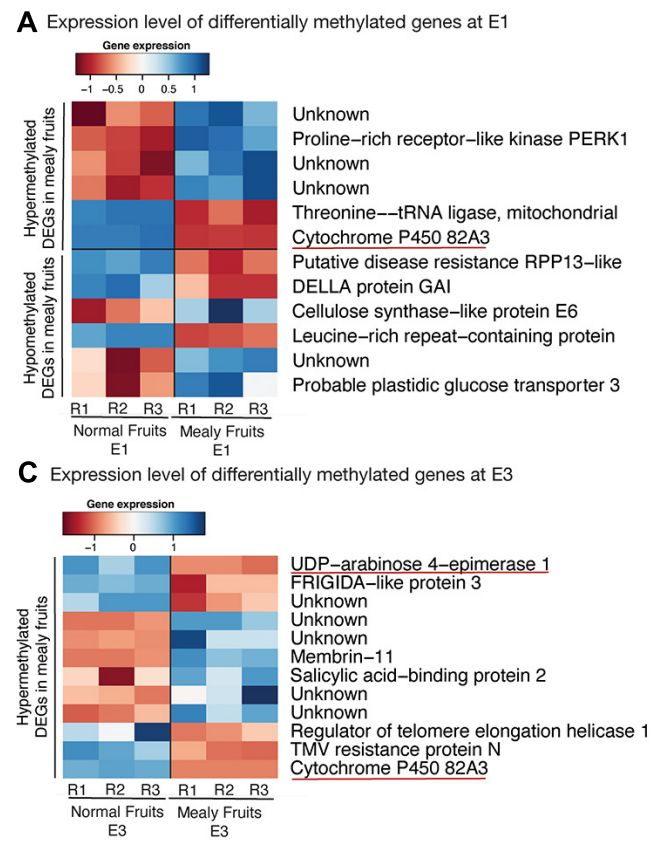
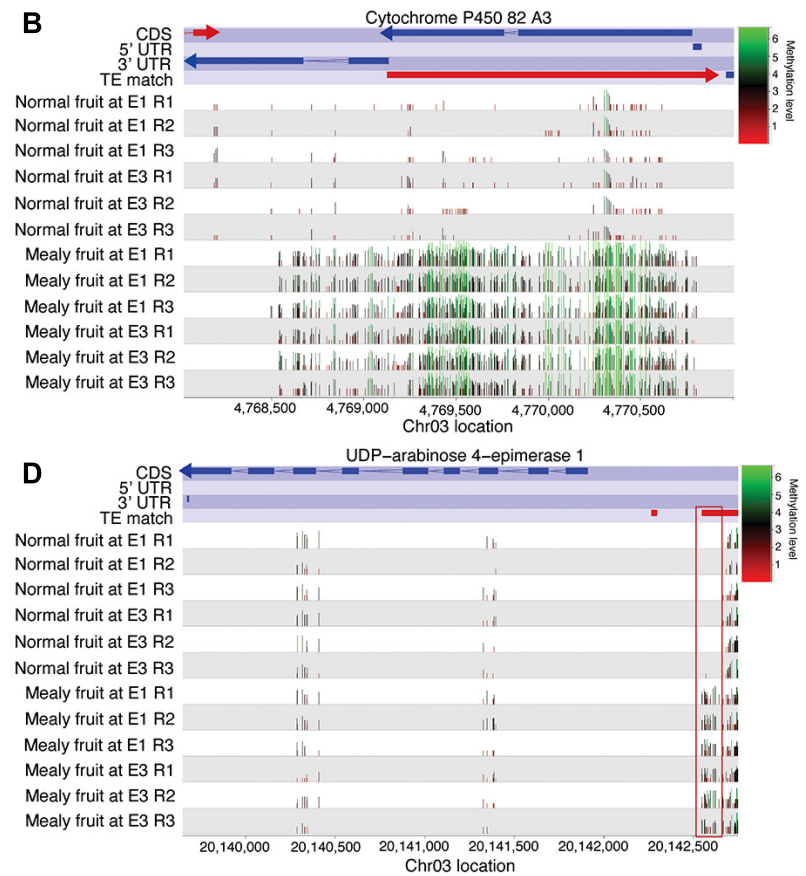

FIGURE 6 | Differentially expressed genes between normal and mealy fruits associated with changes in DNA methylation. (A) Expression levels of DEGs overlapping with DMRs between normal and mealy fruits at E1. DEGs are grouped as hypermethylated and hypomethylated. (B) Cytosine methylation level of a hypermethylated region in mealy fruits compared to normal fruits. The DMR of approximately 2.500 bp is located within the coding region of a CYP450 $82 A 3$ (Prupe.3G066200) gene. Color key indicates methylation levels where green represents high levels and red, low levels. (C) Expression levels of DEGs overlapping with hypermethylated regions between normal and mealy fruits at E3. (D) DNA methylation level of a hypermethylated region in mealy fruits compared to normal fruits. The DMR of approximately $120 \mathrm{bp}$ is located in the promoter region of an UDP-ARABINOSE 4-EPIMERASE1 (Prupe.3G187800) gene.

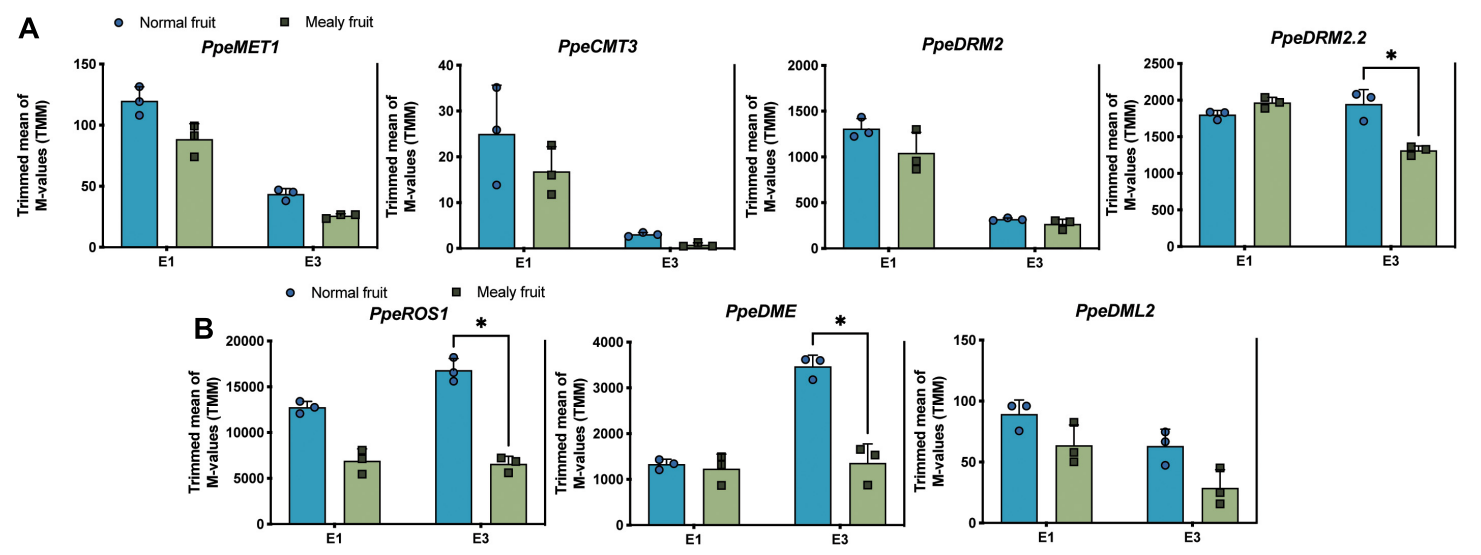

FIGURE 7 | Expression levels of four putative DNA methyltransferases and three 5-mC glycosylases in normal and mealy P.persica fruits. Transcript levels of (A) methyltransferases MET1 (Prupe.7G183100), CMT3 (Prupe.6G011600), DRM2 (Prupe.8G038800), and DRM2.2 (Prupe.6G322700). (B) Transcript levels of 5-mC glycosylases ROS1 (Prupe.7G118000), DME (Prupe.7G005000), and DML2 (Prupe.6G119100). Transcripts were analyzed by RNA-Seq using three biological replicates. Asterisks represent significant differences ( $p$-value $\leq 0.05)$ between normal and mealy fruits using a Two-Way ANOVA test. Error bars represent standard deviation (SD).

(Lurie and Crisosto, 2005; Pegoraro et al., 2015). On the other hand, a gene showing a decrease of methylation in mealy fruits is PECTINESTERASE 68, a gene involved with cell wall metabolism. In this case, an alteration in the activity of polygalacturonases and pectinesterases can lead to the accumulation of gel-forming pectic compounds in the apoplast, retaining free water and resulting in mealy texture (Lurie et al., 2003; Brummell et al., 2004).

At E3 stage, the sugar transporter SWEET2 presented increased methylation levels in mealy fruits, which could be associated with cold storage. Although the accumulation of sugars in peach fruits is an important trait for consumers, they 
also play an important role for cold tolerance by acting as osmolytes and protecting cell membranes (Ding et al., 2019).

\section{Distribution of Global DNA Methylation Patterns Is Related to Transposable Elements and Genetic Variance}

The distribution and level of DNA methylation patterns are important factors for gene regulation and to avoid proliferation of TEs. For instance, gene body methylation is associated with CpG context and usually occurs within exons, but not in TSS and TES (Takuno and Gaut, 2013). According to the same study, methylated genes within their exons at $\mathrm{CpG}$ context, are constitutively expressed, however, $\mathrm{CHG}$ and $\mathrm{CHH}$ methylation tend to be lower within transcribed regions. Coincident with this, the methylation levels at $\mathrm{CHG}$ and $\mathrm{CHH}$ were lower within genes of peach, while methylation at $\mathrm{CpG}$ in transcribed regions was higher.

On the other hand, DNA methylation at gene promoters is associated with silencing by inhibiting the binding of transcription factors (Zhang et al., 2018). DNA methylation in non-coding regions can be consequence of nearby transposons and other repeats, which might be the case of peach, where methylation levels within TEs and neighbor regions is variable. Moreover, TEs respond to environmental stimuli. For example, in A. thaliana the repression of VERNALIZATION INSENSITIVE 3 (VIN3) in the absence of cold is contributed by a TE located at the promoter region (Kim et al., 2010). In our study, the presence of TEs in mealy and normal fruits may generate a dynamic profile of DNA methylations between siblings, which in turn could alter gene regulation nearby. In fact, TEs represent a $29.6 \%$ of the peach genome, whereas a $7.54 \%$ of the genome are uncharacterized repeats (Verde et al., 2013).

The identification of DMRs between E3 and E1 stages in normal and mealy fruits showed that differences in the methylation level in response to cold storage occur mainly upstream and downstream of genes in $\mathrm{CHH}$ cytosines, being hypermethylated at E3 in both siblings, but with an increased number of DMRs in mealy fruits (Figure 3C). Several studies have indicated $\mathrm{CHH}$ methylation as a response to abiotic stress, suggesting a role for transient $\mathrm{CHH}$ methylation in response to environmental stimuli. For instance, during chilling accumulation in sweet cherry (Prunus avium), floral buds showed an increase in $\mathrm{CHH}$ methylation in genes associated with cold signaling, oxidation-reduction process, phenylpropanoid, and lipid metabolism (Rothkegel et al., 2020).

On the other hand, comparisons between mealy and normal fruits also showed hypermethylations of the $\mathrm{CHH}$ context in mealy fruits even before cold storage at E1, suggesting that genetic variance between siblings could result in increased DNA methylations in susceptible fruits. A GO analysis to identify which processes may be related with these DMRs in mealy fruits revealed molecular functions coincident with a metabolic response that is different in mealy fruits regarding to normal fruits. Regarding this, a previous proteomic analysis of peach fruits has showed that processes like ROS metabolism and cellular homeostasis are affected after cold storage (Nilo et al.,
2010). In the same study, a decreased accumulation of catalases and ferritins was observed in cold stored fruits, suggesting an interaction between iron homeostasis and ROS levels.

\section{Genes Associated With Ethylene Signaling, Cell Wall Modification, Lipid Metabolism, Oxidative Stress, and Iron Homeostasis Are Differentially Expressed in Mealy Fruits}

Responses to low temperatures involve transcriptomic and metabolic reprogramming. At the transcriptomic level, PCA and the number of DEGs showed that gene expression is affected not only by the genotype of each individual, but also by the fruit condition (E1 and E3). Moreover, most DEGs were obtained between E1 and E3 rather than in mealy vs. normal fruits as observed in methylome data. This difference in gene expression may be due to an energetic reprogramming in order to establish a new metabolic homeostasis for acclimation under cold conditions, which is regulated by several factors, such as signaling pathways and transcription factors (Fürtauer et al., 2019).

Regarding overrepresented pathways, ethylene signaling is essential for a series of developmental processes, such as growth and senescence, and responses to abiotic and biotic stress. In peach, we identify the ethylene response factors ERF109, ERF017, and $D R E B 1 D$ with higher expression in mealy fruits at E1 stage when compared to $\mathrm{E} 3$, while $E R F 1 B$ was downregulated at $\mathrm{E} 1$ and E3 (Figure 8), coincident with previous studies that suggested that $\mathrm{CI}$ is associated with a decreased ethylene production (Wang et al., 2017; Nilo-Poyanco et al., 2018).

Another determinant factor for the development of mealiness is the cell wall, where initial studies have found an association between mealiness and the altered activity of pectin methylesterases (PME) and endo-polygalacturonases during cold storage, resulting in higher levels of soluble pectins and mealy texture (Buescher and Furmanski, 1978; Ben-Arie and Sonego, 1980; Lurie et al., 1994). Concomitantly, in mealy fruits at E1 we identify a GALACTURONOSYLTRANSFERASElike 10 (GAUT10) showing higher expression (Figure 8). Afterward at E3, XYLOGLUCAN XTH8 and a probable POLYGALACTURONASE $(P G)$ presented an increased expression, while a CELL WALL-ASSOCIATED RECEPTOR KINASE 2 WAK2 was downregulated, suggesting an altered cell wall metabolism during cold storage.

As one of the first barriers to the environment, the cell membrane is important to integrate the environmental signal into the cell. In the case of low temperatures, a decrease in membrane fluidity correlates with the proportion of desaturated fatty acids, affecting the metabolism of lipids (Martiniere et al., 2011). Moreover, in A. thaliana, the levels of polyunsaturated fatty acids are pivotal for survival to low temperatures $\left(<12^{\circ} \mathrm{C}\right)$ (Miquel et al., 1993). In peach, we have found that LTPG1, associated with lipid transport, is overexpressed in mealy fruits after cold storage (Figure 8). While a GDSL esterase/lipase and a O-ACYLTRANSFERASE WSD1 are downregulated in mealy fruits in both conditions, indicating that the lipid metabolism is differentially regulated probably due to the genetic background 


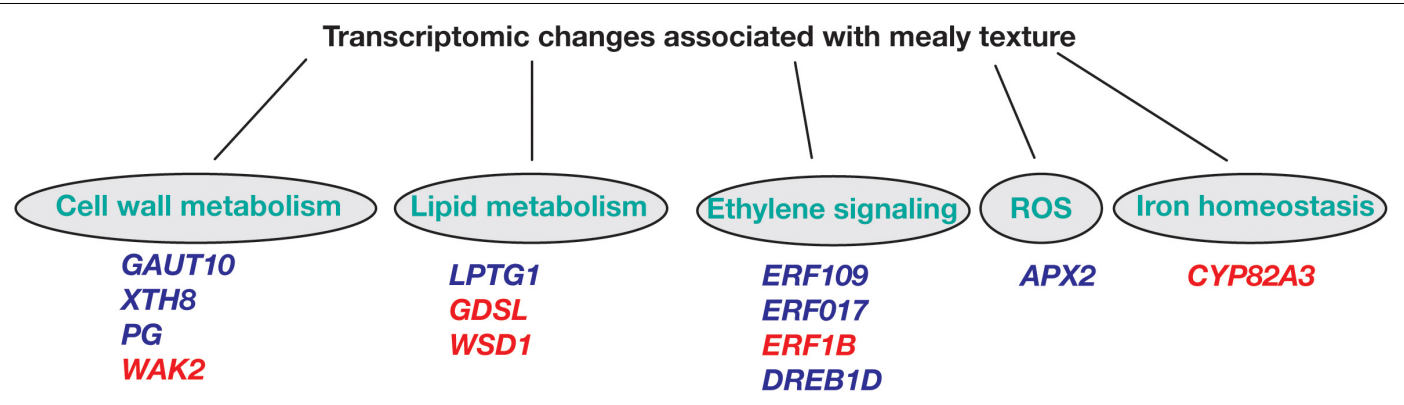

FIGURE 8 | Hypothetical scheme of differentially expressed genes between mealy and normal fruits associated to metabolic pathways leading to mealiness in P. persica fruits. Biological processes of cell wall metabolism, lipid metabolism, ethylene signaling, ROS (reactive oxygen species) and iron homeostasis are represented. Genes highlighted in blue are overexpressed, while red colored genes are downregulated in mealy fruits. GAUT10:

GALACTURONOSYLTRANSFERASE-like 10; XTH8: XYLOGLUCAN ENDOTRANSGLUCOSYLASE/HYDROLASE PROTEIN 8; PG: POLYGALACTURONASE; WAK2: WALL-ASSOCIATED RECEPTOR KINASE 2; LPTG1: NON-SPECIFIC LIPID TRANSFER PROTEIN GPI-ANCHORED 1; GDSL ESTERASE/lipaSe; WSD1:

O-ACYLTRANSFERASE; ERF: ETHYLENE-RESPONSIVE FACTOR; DREB1D: DEHYDRATION-RESPONSIVE ELEMENT BINDING 1D; APX2: L-ASCORBATE PEROXIDASE 2; CYP82A3: CYTOCHROME P450 82 A3.

of each individual since these changes occurred before cold storage. In fact, a recent study using the same peach individuals has identified 119 metabolites and 189 lipids with significant differences in abundance between juicy and mealy fruits, where lipids were proposed as biomarkers for mealiness at E1 and E3 stages (Lillo-Carmona et al., 2020).

Regarding oxidative stress, the signaling of ROS is associated with biotic and abiotic responses. Usually, an abiotic stress like low temperatures increase the levels of ROS because of a lower stability of scavenging enzymes (Ding et al., 2019). Some of these scavenging enzymes correspond to superoxide dismutase, ascorbate peroxidase, catalase, and glutathione peroxidase (Müller and Munné-Bosch, 2015). In this study, we identify a PEROXIDASE APX2 that is highly expressed in mealy fruits before and after cold storage (Figure 8), indicating that susceptible fruits might generate lower ROS levels and thus, a lower signal to induce a cold response.

The connection between ROS and iron homeostasis has also been reported, where hydrogen peroxide $\left(\mathrm{H}_{2} \mathrm{O}_{2}\right)$ molecules react with $\mathrm{Fe}^{2+}$ to generate hydroxyl radicals, which attack pectin polysaccharides, decreasing mealiness susceptibility (Airianah et al., 2016; Choudhury et al., 2016). Furthermore, we have found that a CYP82A3 gene associated with iron deficiency is silenced in mealy fruits (Figure 8) and thus, we hypothesize that low levels of $\mathrm{Fe}^{2+}$ due to CYP82A3 silencing and low levels of ROS due to high APX2 expression, could result in lower hydroxyl radicals, promoting a favorable environment for pectin accumulation and mealiness development.

\section{The Downregulation of CYP82A3 and UDP-ARABINOSE 4 in Mealy Fruits Is Accompanied by Higher Methylation Levels and the Presence of a TE}

The superfamily of CYTOCHROME P450 has an important role in promoting plant growth, development, and stress responses with detoxification pathways (Xu et al., 2015). Regarding development, CYP 450s are involved in several metabolic pathways, such as cytokinin and flavonoid biosynthesis, and iron deficiency (Kruse et al., 2008; Berim and Gang, 2013). As previously mentioned, we have found that a putative CYP82A3 is not expressed in mealy fruits, which is associated with its hypermethylation. Moreover, this hypermethylation coincides with the localization of a TE in the opposite strand, suggesting that the presence of the TE might be responsible for an increase in methylation levels, affecting gene expression.

Another downregulated gene in mealy fruits is UDP$A R A B I N O D E$ 4. L-arabinose (Ara) in plants is an important component of glycoproteins, flavonoids, signaling peptides and cell wall polysaccharides, participating in cell wall biosynthesis (Rautengarten et al., 2017). In this sense, an imbalance of Ara affects directly the composition of the cell wall and may also be involved in mealiness texture, where a decreased cellto-cell adhesion causes cell separation instead of cell rupture during chewing (Brummell et al., 2004). In our study, the downregulation of UDP-ARABINOSE 4 EPIMERASE 1 is associated with an increase in the methylation level upstream. The encoded protein catalyzes the 4-epimerization of UDP- $D$-Xyl to $\mathrm{UDP}_{-}{ }_{-}$-Ara (Burget et al., 2003), suggesting that the levels of xylose could also be affected in mealy fruits. Interestingly, this hypermethylation co-localized with a TE match, which may affect gene expression in a similar way than CYP82A3. Altogether, these results highlight that genetic variability due to TEs and repetitive elements results in increased DNA methylation levels in mealy fruits before cold storage, which could affect gene expression and tolerance during cold storage.

\section{DNA Glycosylases Are Downregulated in Mealy Fruits, Contributing With an Increase in DNA Methylations}

DNA methyltransferases and glycosylases are key factors for the establishment and maintenance of methylation patterns. Methylation in the $\mathrm{CpG}$ context is the most abundant and is maintained during DNA replication by DNA METHYLTRANSFERASE 1 (MET1) (Finnegan et al., 1996). 
While methylation in the CHG context is maintained by a reinforcing loop involving CHROMOMETHYLASE 3 (CMT3) and histone marks (H3K9) (Du et al., 2012). Methylation in $\mathrm{CHH}$ occurs de novo and is maintained by DOMAINS REARRANGED METHYLTRANSFERASE 2 (DRM2), together with non-coding RNAs in a pathway known as RNA-directed DNA Methylation (RdDM) (Law and Jacobsen, 2010). In peach we have found four homologous to these DNA methyltransferases, PpeMET1, PpeCMT3, PpeDRM2, and PpeDRM2.2. The transcript levels for all these genes have shown a decrease in expression at E3 stage in normal and mealy fruits, indicating that the regulation of these genes is affected by low temperatures in peach fruits.

On the other hand, DNA demethylation involves the participation of glycosylases REPRESSOR OF SILENCING 1 (ROS1), DEMETER (DME) and DEMETER-like (DML), which act during DNA reparation through a process of base excision, removing methylated cytosines (Gong et al., 2002; Choi et al., 2002; Ortega-Galisteo et al., 2008). In peach, we have identified PpeROS1, PpeDME, and PpeDML2, whose expression increased after cold storage only in normal fruits, indicating a higher control of DNA methylations. On the contrary, mealy fruits were associated with higher levels of DNA methylations, possibly due to a lower expression of DNA glycosylases. These results are concomitant with the key role described for DNA demethylation during fruit ripening by Lang et al. (2017), where mutant tomato plants for a ROS1 homolog gene failed to ripen because ripening genes were silenced by DNA methylations.

\section{CONCLUSION}

In conclusion, in this study we obtained different DNA methylation patterns between normal and mealy fruits, showing higher methylation levels in mealy fruits, particularly in the $\mathrm{CHH}$ context. Moreover, these differences in methylation appears to be mostly influenced by genetic differences between siblings (e.g., TEs and repetitive elements), coinciding with previous QTL studies. When integrating RNA-Seq data, we found that CYP82A3 and UDP-ARABINOSE 4 EPIMERASE 1 are downregulated in mealy fruits, coinciding with the presence of TEs, possibly affecting iron homeostasis and cell wall metabolism. From gene expression data, a lower expression of DNA glycosylases during cold storage could contribute with higher methylation levels in mealy fruits. Taken together, this study provides an additional layer of information to better understand the complex phenotype of mealiness. Moreover, the

\section{REFERENCES}

Airianah, O. B., Vreeburg, R. A. M., and Fry, S. C. (2016). Pectic polysaccharides are attacked by hydroxyl radicals in ripening fruit: evidence from a fluorescent fingerprinting method. Ann. Bot. 117, 441-455. doi: 10.1093/aob/mcv192

Ben-Arie, R., and Lavee, S. (1971). Pectic changes occurring in Elberta peaches suffering from woolly breakdown. Phytochemistry 10, 531-538. doi: 10.1016/ S0031-9422(00)94693-4

Ben-Arie, R., and Sonego, L. (1980). Pectolytic enzyme activity involved in woolly breakdown of stored peaches. patterns of DNA methylation and expression levels of CYP82A3 and UDP-ARABINOSE 4 could be further studied as novel markers for their use, together with molecular markers, in order to support peach breeding programs.

\section{DATA AVAILABILITY STATEMENT}

The datasets presented in this study can be found in online repositories. The names of the repository/repositories and accession number(s) can be found below: https://www.ncbi.nlm. nih.gov/genbank/, PRJNA705001; PRJNA705416.

\section{AUTHOR CONTRIBUTIONS}

$\mathrm{CM}, \mathrm{RC}-\mathrm{V}$, and $\mathrm{KR}$ designed the research. AE, AR, and VL-C conducted field work and phenotyping. KR generated MethylCSeq libraries. AE and KR contributed with bioinformatic analysis of MethylC-Seq. DS contributed with RNA-seq and qPCR experiments, while $\mathrm{AR}, \mathrm{AE}$, and $\mathrm{KR}$ performed bioinformatic analysis of RNA-seq. CM conducted statistical analysis and KR wrote the original manuscript. KR, CM, VL-C, DS, and RC-V edited and reviewed the original manuscript. All authors read and approved the submitted version.

\section{FUNDING}

This study was funded by Fondo Nacional de Desarrollo Científico y Tecnológico (ANID-FONDECYT 1200804), a Ph.D. national grant (ANID-PFCHA/Doctorado Nacional 201721170365), and ANID-FONDECYT POSTDOCTORADO 20183180306.

\section{ACKNOWLEDGMENTS}

We want to thank INIA Rayentué for giving us access to the $\mathrm{V} x$ $\mathrm{V}$ segregant population from their fields.

\section{SUPPLEMENTARY MATERIAL}

The Supplementary Material for this article can be found online at: https://www.frontiersin.org/articles/10.3389/fpls.2021. 684130/full\#supplementary-material

Phytochemistry 19, 2553-2555. doi: 10.1016/S0031-9422(00) 83917-5

Berim, A., and Gang, D. R. (2013). The roles of a flavone-6hydroxylase and 7-O-demethylation in the flavone biosynthetic network of sweet basil. J. Biol. Chem. 288, 1795-1805. doi: 10.1074/jbc.M112.420448

Brummell, D. A., Dal Cin, V., Lurie, S., Crisosto, C. H., and Labavitch, J. M. (2004). Cell wall metabolism during the development of chilling injury in coldstored peach fruit: association of mealiness with arrested disassembly of cell wall pectins. J. Exp. Bot. 55, 2041-2052. doi: 10.1093/jxb/erh228 
Buescher, R. W., and Furmanski, R. J. (1978). Role of pectinesterase and polygalacturonase in the formation of woolliness in peaches. J. Food Sci. 43, 264-266. doi: 10.1111/j.1365-2621.1978.tb09788.x

Burget, E. G., Verma, R., Mølhøj, M., and Reiter, W. D. (2003). The biosynthesis of L-Arabinose in plants. Plant Cell 15, 523-531. doi: 10.1105/tpc.008425

Campos-Vargas, R., Becerra, O., Baeza-Yates, R., Cambiazo, V., González, M., Meisel, L., et al. (2006). Seasonal variation in the development of chilling injury in 'O'Henry' peaches. Sci. Hortic. 110, 79-83. doi: $10.1016 /$ j.scienta.2006.06.019

Cantín, C. M., Crisosto, C. H., Ogundiwin, E. A., Gradziel, T., Torrents, J., Moreno, M. A., et al. (2010). Chilling injury susceptibility in an intra-specific peach [Prunus persica (L.) Bastch] progeny. Postharvest Biol. Technol. 58, 79-87. doi: 10.1016/j.postharvbio.2010.06.002

Choi, Y., Gehring, M., Johnson, L., Hannon, M., Harada, J. J., Goldberg, R. B., et al. (2002). DEMETER, a DNA glycosylase domain protein, is required for endosperm gene imprinting and seed viability in Arabidopsis. Cell 110, 33-42. doi: 10.1016/s0092-8674(02)00807-3

Choudhury, F. K., Rivero, R. M., Blumwald, E., and Mittler, R. (2016). Reactive oxygen species, abiotic stress and stress combination. Plant J. 90, 856-867. doi: $10.1111 /$ tpj.13299

Crisosto, C. H., Mitchell, F. G., and Ju, Z. G. (1999). Susceptibility to chilling injury of peach, nectarine, and plum cultivars grown in California. HortScience 34, 1116-1118. doi: 10.21273/HORTSCI.34.6.1116

Daccord, N., Celton, J. M., Linsmith, G., Becker, C., Choisne, N., Schijlen, E., et al. (2017). High-quality de novo assembly of the apple genome and methylome dynamics of early fruit development. Nat. Genet. 49, 1099-1106. doi: 10.1038/ ng. 3886

Ding, Y., Shi, Y., and Yang, S. (2019). Advances and challenges in uncovering cold tolerance regulatory mechanisms in plants. New Phytol. 222, 1690-1704. doi: $10.1111 / \mathrm{nph} .15696$

Dobin, A., Davis, C. A., Schlesinger, F., Drenkow, J., Zaleski, C., Jha, S., et al. (2013). STAR: ultrafast universal RNA-seq aligner. Bioinformatics 29, 15-21. doi: 10.1093/bioinformatics/bts635

Du, J., Zhong, X., Bernatavichute, Y. V., Stroud, H., Feng, S., Caro, E., et al. (2012). Dual binding of chromomethylase domains to H3K9me2-containing nucleosomes directs DNA methylation in plants. Cell 151, 167-180. doi: 10. 1016/j.cell.2012.07.034

Finnegan, E. J., Peacock, W. J., and Dennis, E. S. (1996). Reduced DNA methylation in Arabidopsis thaliana results in abnormal plant development. Proc. Natl. Acad. Sci. U.S.A. 93, 8449-8454. doi: 10.1073/pnas.93.16.8449

Fürtauer, L., Weiszmann, J., Weckwerth, W., and Nägele, T. (2019). Dynamics of plant metabolism during cold acclimation. Int. J. Mol. Sci. 20:5411. doi: 10.3390/ijms20215411

García-Gómez, B. E., Salazar, J. A., Nicolás-Almansa, M., Razi, M., Rubio, M., Ruiz, D., et al. (2021). Molecular bases of fruit quality in Prunus species: an integrated genomic, transcriptomic, and metabolic review with a breeding perspective. Int. J. Mol. Sci. 22, 333. doi: 10.3390/ijms22010333

Gong, Z., Morales-Ruiz, T., Ariza, R. R., Roldán-Arjona, T., David, L., and Zhu, J. K. (2002). ROS1, a repressor of transcriptional gene silencing in Arabidopsis, encodes a DNA glycosylase/lyase. Cell 111, 803-814. doi: 10.1016/S00928674(02)01133-9

Infante, R., Meneses, C., and Crisosto, C. H. (2009). Preconditioning treatment maintains taste characteristic perception of ripe 'September Sun' peach following cold storage. Int. J. Food Sci. Technol. 44, 1011-1016. doi: 10.1111/ j.1365-2621.2008.01864.x

Kan, J., Wang, H., and Jin, C. (2011). Changes of reactive oxygen species and related enzymes in mitochondrial respiration during storage of harvested peach fruits. Agric. Sci. China 10, 149-158. doi: 10.1016/S1671-2927(11)60317-9

Kim, D. H., Zografos, B. R., and Sung, S. (2010). Vernalizationmediated VIN3 induction overcomes the LIKE-HETEROCHROMATIN PROTEIN1/POLYCOMB REPRESSION COMPLEX2-mediated epigenetic repression. Plant Physiol. 154, 949-957. doi: 10.1104/pp.110.161083

King, G. A., Henderson, K. G., and Lill, R. E. (1989). Ultrastructural changes in the nectarine cell wall accompanying ripening and storage in a chillingresistant and chilling-sensitive cultivar. N. Z. J. Crop Hortic. Sci. 17, 337-344. doi: 10.1080/01140671.1989.10428054
Kishore, K., de Pretis, S., Lister, R., Morelli, M. J., Bianchi, V., Amati, B., et al. (2015). MethylPipe and compEpiTools: a suite of R packages for the integrative analysis of epigenomics data. BMC Bioinformatics 16:313. doi: 10.1186/s12859015-0742-6

Krueger, F., and Andrews, S. R. (2011). Bismark: a flexible aligner and methylation caller for bisulfite-seq applications. Bioinformatics 27, 1571-1572. doi: 10.1093/ bioinformatics/btr167

Kruse, T., Ho, K., Yoo, H. D., Johnson, T., Hippely, M., Park, J. H., et al. (2008). In planta biocatalysis screen of P450s identifies 8-methoxypsoralen as a substrate for the CYP82C subfamily, yielding original chemical structures. Chem. Biol. 15, 149-156. doi: 10.1016/j.chembiol.2008.01.008

Lang, Z., Wang, Y., Tang, K., Tang, D., Datsenka, T., Cheng, J., et al. (2017). Critical roles of DNA demethylation in the activation of ripening-induced genes and inhibition of ripening-repressed genes in tomato fruits. Proc. Natl. Acad. Sci. U.S.A. 114, E4511-E4519. doi: 10.1073/pnas.1705233114

Law, J. A., and Jacobsen, S. E. (2010). Establishing, maintaining and modifying DNA methylation patterns in plants and animals. Nat. Rev. Genet. 11, 204-220. doi: $10.1038 / \operatorname{nrg} 2719$

Lillo-Carmona, V., Espinoza, A., Rothkegel, K., Rubilar, M., Nilo-Poyanco, R., Pedreschi, R., et al. (2020). Identification of metabolite and lipid profiles in a segregating peach population associated with mealiness in Prunus persica (L.) Batsch. Metabolites 10:154. doi: 10.3390/metabo10040154

Lister, R., O’Malley, R. C., Tonti-Filippini, J., Gregory, B. D., Berry, C. C., Millar, A. H., et al. (2008). Highly integrated single-base resolution maps of the epigenome in Arabidopsis. Cell 133, 523-536. doi: 10.1016/j.cell.2008.03.029

Lurie, S., and Crisosto, C. H. (2005). Chilling injury in peach and nectarine. Postharvest Biol. Technol. 37, 195-208. doi: 10.1016/j.postharvbio.2005.04.012

Lurie, S., Levin, A., Greve, L. C., and Labavitch, J. M. (1994). Pectic polymer changes in nectarines during normal and abnormal ripening. Phytochemistry 36, 11-17. doi: 10.1016/S0031-9422(00)97003-1

Lurie, S., Zhou, H. W., Lers, A., Sonego, L., Alexandrov, S., and Shomer, I. (2003). Study of pectin esterase and changes in pectin methylation during normal and abnormal peach ripening. Physiol. Plant 119, 287-294. doi: 10.1034/j.13993054.2003.00178.x

Manning, K., Tör, M., Poole, M., Hong, Y., Thompson, A. J., King, G. J., et al. (2006). A naturally occurring epigenetic mutation in a gene encoding an SBPbox transcription factor inhibits tomato fruit ripening. Nat. Genet. 38, 948-952. doi: $10.1038 /$ ng1841

Martiniere, A., Shvedunova, M., Thomson, A. J., Evans, N. H., Penfield, S., Runions, J., et al. (2011). Homeostasis of plasma membrane viscosity in fluctuating temperatures. New Phytol. 192, 328-337. doi: 10.1111/j.1469-8137.2011. 03821.x

Meisel, L., Fonseca, B., González, S., Baeza-Yates, R., Cambiazo, V., Campos, R., et al. (2005). A rapid and efficient method for purifying high quality total RNA from peaches (Prunus persica) for functional genomics analyses. Biol. Res. 38, 83-88. doi: 10.4067/s0716-97602005000100010

Miquel, M., James, D. Jr., Dooner, H., and Browse, J. (1993). Arabidopsis requires polyunsaturated lipids for low-temperature survival. Proc. Natl. Acad. Sci. U.S.A. 90, 6208-6212. doi: 10.1073/pnas.90.13.6208

Monti, L. L., Bustamante, C. A., Budde, C. O., Gabilondo, J., Müller, G. L., Lara, M. V., et al. (2019). Metabolomic and proteomic profiling of Spring Lady peach fruit with contrasting woolliness phenotype reveals carbon oxidative processes and proteome reconfiguration in chilling-injured fruit. Postharvest Biol. Technol. 151, 142-151. doi: 10.1016/j.postharvbio.2019.02.007

Müller, M., and Munné-Bosch, S. (2015). Ethylene response factors: a key regulatory hub in hormone and stress signaling. Plant Physiol. 169, 32-41. doi: 10.1104/pp.15.00677

Nilo, R., Saffie, C., Lilley, K., Baeza-Yates, R., Cambiazo, V., Campos-Vargas, R., et al. (2010). Proteomic analysis of peach fruit mesocarp softening and chilling injury using difference gel electrophoresis (DIGE). BMC Genomics 11:43. doi: 10.1186/1471-2164-11-43

Nilo-Poyanco, R., Vizoso, P., Sanhueza, D., Balic, I., Meneses, C., Orellana, L. A., et al. (2018). A Prunus persica genome-wide RNA-seq approach uncovers major differences in the transcriptome among chilling injury sensitive and non-sensitive varieties. Physiol. Plant. 166, 772-793. doi: 10.1111/ppl.12831

Nuñez-Lillo, G., Balladares, C., Pavez, C., Urra, C., Sanhueza, D., Vendramin, E., et al. (2019). High-density genetic map and QTL analysis of soluble solid 
content, maturity date, and mealiness in peach using genotyping by sequencing. Sci. Hortic. 257:108734. doi: 10.1016/j.scienta.2019.108734

Nuñez-Lillo, G., Cifuentes-Esquivel, A., Troggio, M., Micheletti, D., Infante, R., Campos-Vargas, R., et al. (2015). Identification of candidate genes associated with mealiness and maturity date in peach [Prunus persica (L.) Batsch] using QTL analysis and deep sequencing. Tree Genet. Genomes 11:86. doi: 10.1007/ s11295-015-0911-9

Ortega-Galisteo, A. P., Morales-Ruiz, T., Ariza, R. R., and Roldán-Arjona, T. (2008). Arabidopsis DEMETER-LIKE proteins DML2 and DML3 are required for appropriate distribution of DNA methylation marks. Plant Mol. Biol. 67, 671-681. doi: 10.1007/s11103-008-9346-0

Peace, C. P., Crisosto, C. H., and Gradziel, T. M. (2005). Endopolygalacturonase: a candidate gene for freestone and melting flesh in peach. Mol. Breed. 16, 21-31. doi: 10.1007/s11032-005-0828-3

Pegoraro, C., Tadiello, A., Girardi, C. L., Chaves, F. C., Quecini, V., Costa de Oliveira, A., et al. (2015). Transcriptional regulatory networks controlling woolliness in peach in response to preharvest gibberellin application and cold storage. BMC Plant Biol. 15:279. doi: 10.1186/s12870-015-0659-2

Pons, C., Marti, C., Forment, J., Crisosto, C. H., Dandekar, A. M., and Granell, A. (2014). A bulk segregant gene expression analysis of a peach population reveals components of the underlysing mechanism of the fruit cold response. PLoS One 9:e90706. doi: 10.1371/journal.pone.0090706

Pons, C., Marti, C., Forment, J., Crisosto, C. H., Dandekar, A. M., and Granell, A. (2016). A genetic genomics-expression approach reveals components of the molecular mechanisms beyond the cell wall that underlie peach fruit woolliness due to cold storage. Plant Mol. Biol. 92, 483-503. doi: 10.1007/s11103-0160526-z

Prudencio, A. S., Werner, O., Martínez-García, P. J., Dicenta, F., Ros, R. M., and Martínez-Gómez, P. (2018). DNA methylation analysis of dormancy release in almond (Prunus dulcis) flower buds using epi-genotyping by sequencing. Int. J. Mol. Sci. 19:3542. doi: 10.3390/ijms19113542

Puig, C. P., Dagar, A., Ibanez, C. M., Singh, V., Crisosto, C. H., Friedman, H., et al. (2015). Pre-symptomatic transcriptome changes during cold storage of chilling sensitive and resistant peach cultivars to elucidate chilling injury mechanisms. BMC Genomics 16:245. doi: 10.1186/s12864-015-1395-6

Rautengarten, C., Birdseye, D., Pattathil, S., McFarlane, H. E., Saez-Aguayo, S., Orellana, A., et al. (2017). The elaborate route for UDP-arabinose delivery into the Golgi of plants. Proc. Natl. Acad. Sci. U.S.A. 114, 4261-4266. doi: 10.1073/pnas.1701894114

Robinson, M. D., McCarthy, D. J., and Smyth, G. K. (2010). EdgeR: a Bioconductor package for differential expression analysis of digital gene expression data. Bioinformatics 26, 139-140. doi: 10.1093/bioinformatics/btp616

Rothkegel, K., Sánchez, E., Montes, C., Greve, M., Tapia, S., Bravo, S., et al. (2017). DNA methylation and small interference RNAs participate in the regulation of MADS-box genes involved in dormancy in sweet cherry (Prunus avium L.). Tree Physiol. 37, 1739-1751. doi: 10.1093/treephys/ tpx055
Rothkegel, K., Sandoval, P., Soto, E., Lissette, U., Riveros, A., Lillo, V., et al. (2020). Dormant but active: chilling accumulation modulates the epigenome and transcriptome of Prunus avium during bud dormancy. Front. Plant Sci. 11:1115. doi: 10.3389/fpls.2020.01115

Su, Y., Bai, X., Yang, W., Wang, W., Chen, Z., Ma, J., et al. (2018). Single-baseresolution methylomes of Populus euphratica reveal the association between DNA methylation and salt stress. Tree Genet. Genomes 14:86. https://link. springer.com/article/10.1007/s11295-018-1298-1

Takuno, S., and Gaut, B. S. (2013). Gene body methylation is conserved between plant orthologs and is of evolutionary consequence. Proc. Natl. Acad. Sci. U.S.A. 110, 1797-1802. doi: 10.1073/pnas.1215380110

Tong, Z., Gao, Z., Wang, F., Zhou, J., and Zhang, Z. (2009). Selection of reliable reference genes for gene expression studies in peach using real-time PCR. BMC Mol. Biol. 10:71. doi: 10.1186/1471-2199-10-71

Verde, I., Abbot, A., Scalabrn, S., Jung, S., Shu, S., Marroni, F., et al. (2013). The high-quality draft genome of peach (Prunus persica) identifies unique patterns of genetic diversity, domestication and genome evolution. Nat. Genet. 45, 487-494. doi: 10.1038/ng.2586

Verde, I., Jenkins, J., Dondini, L., Micali, S., Pagliarani, G., Vendramin, E., et al. (2017). The Peach v2.0 release: high-resolution linkage mapping and deep resequencing improve chromosome-scale assembly and contiguity. BMC Genomics 18:225. doi: 10.1186/s12864-017-3606-9

Wang, K., Yin, X. R., Zhang, B., Grierson, D., Xu, C. J., and Chen, K. S. (2017). Transcriptomic and metabolic analyses provide new insights into chilling injury in peach fruit. Plant Cell Environ. 40, 1531-1551. doi: 10.1111/pce.12951

Xu, J., Wang, X., and Guo, W. (2015). The cytochrome P450 superfamily: key players in plant development and defense. J. Integr. Agric. 14, 1673-1686. doi: 10.1016/s2095-3119(14)60980-1

Zhang, H., Lang, Z., and Zhu, J. K. (2018). Dynamics and function of DNA methylation in plants. Nat. Rev. Mol. Cell Biol. 19, 489-506. doi: 10.1038/ s41580-018-0016-Z

Zhao, Y., Song, C., Brummel, D. A., Qi, S., Lin, Q., and Duan, Y. (2021). Jasmonic acid treatment alleviates chilling injury in peach fruit by promoting sugar and ethylene metabolism. Food Chem. 338:128005. doi: 10.1016/j.foodchem.2020. 128005

Conflict of Interest: The authors declare that the research was conducted in the absence of any commercial or financial relationships that could be construed as a potential conflict of interest.

Copyright $\odot 2021$ Rothkegel, Espinoza, Sanhueza, Lillo-Carmona, Riveros, CamposVargas and Meneses. This is an open-access article distributed under the terms of the Creative Commons Attribution License (CC BY). The use, distribution or reproduction in other forums is permitted, provided the original author(s) and the copyright owner(s) are credited and that the original publication in this journal is cited, in accordance with accepted academic practice. No use, distribution or reproduction is permitted which does not comply with these terms. 